\title{
Intracultural Cognizance of Medicinal Plants of Warangal North Forest Division, Northern Telangana, India
}

\author{
Sateesh Suthari, N. Sreeramulu, K. Omkar, \\ C.S. Reddy, and Vatsavaya S. Raju
}

\section{Research}

\begin{abstract}
Differences in the traditional botanical knowledge of Koya communities inhabiting Eturnagaram Wildlife Sanctuary (Warangal North Forest Division) are investigated. Eighteen villages (16 within the wildlife sanctuary and two outside it) were selected to test the null hypothesis that there exist no cognitive differences among the ethnic inhabitants in their ability to recognize the plants and recall the vernacular names and medicinal uses since they are recipients of the same dry deciduous forest ecosystem services. The Koyas were found to use as medicine 237 species in 66 angiosperm families. Analyses of data gathered from villagers showed that there is significant intracultural diversity in terms of taxonomic groups and growth forms in regard to utilizing the proximate plant resource for their primary healthcare and disease treatment of pets.
\end{abstract}

\section{Introduction}

Biocultural diversity is a source of adaptive capacity since it represents the range of possibilities for humans to sustain communities in dynamic landscapes (Harmon 2002). The survival of a particular community may depend on diversity at local scales (Kassam 2009). Indigenous knowledge systems are therefore increasingly appreciated for their ability to anticipate, recognize, and respond to change (Ruelle \& Kassam 2011, Turner \& Clifton 2009). Moreover, while communities may rely on variation in knowledge to adapt to social and ecological changes, there has been meager attention paid to differences within cultural groups (Ruelle \& Kassam 2011).

It is the common experience of traditional knowledge gatherers all over the globe that the consumption or utilization of non-cultivated plants has declined because younger generations are not learning how to gather them for treating and preventing diseases or ailments in their communities (Ruelle \& Kassam 2011). Therefore, intracultural cognizance should be studied at local, regional, and global scales to determine knowledge loss. There are few studies on intracultural cognizance of ethnic communities in India, let alone Koya community of southern India.

The objectives of this study are: (i) documentation of the medicinal plant knowledge of the Koya communities native to Godavari River Valley; (ii) analyses of the medicinal plants used by Koyas in terms of taxonomic diversity, growth forms, and nativity; and (iii) realization of, if any, intracultural differences in medicinal plant knowledge.

\section{Correspondence}

Sateesh Suthari, Department of Botany, Kakatiya University, Warangal, Andhra Pradesh - 506 009, INDIA. suthari.botany@gmail.com

N. Sreeramulu, Department of Botany, Kakatiya University, Warangal, Andhra Pradesh - 506 009, INDIA. snallella@gmail.com

K. Omkar, PSC \& KVSC Govt. Degree College, Nandyal, Kurnool, Andhra Pradesh - 518 502, INDIA. omkarbot@gmail.com

C.S. Reddy, Forestry \& Ecology Division, National Remote Sensing Centre, Balanagar, Hyderabad, Andhra Pradesh - 500 037, INDIA. drsudhakarreddy@gmail.com

Vatsavaya S. Raju, Department of Botany, Kakatiya University, Warangal, Andhra Pradesh - 506 009, INDIA. rajuvatsavaya@gmail.com

Ethnobotany Research \& Applications 12:211-235 (2014)

Published: 30 June 2014 


\section{Study site}

The North Forest Division of Warangal (Northern Telangana, Andhra Pradesh, India) lies between $17^{\circ} 29^{\prime} 16^{\prime \prime}$ and $18^{\circ} 36^{\prime} 20^{\prime \prime} \mathrm{N}$ and $78^{\circ} 49^{\prime} 49^{\prime \prime}$ and $80^{\circ} 40^{\prime} 13^{\prime \prime} \mathrm{E}$ (Figure 1). The division has a geographical area of $8,687.81 \mathrm{~km}^{2}$ which is $67.6 \%$ of the total area of the district $\left(12,847 \mathrm{~km}^{2}\right)$. Elevation is between 266 and $518 \mathrm{~m}$, with a general SE slope along which surplus waters drain into the river Godavari. The climate is tropical, generally dry with temperature ranging from $15^{\circ} \mathrm{C}$ to $45^{\circ} \mathrm{C}$ and annual rainfall of 1182 $\mathrm{mm}$, received mainly through south-west monsoon. Soils are primarily black cotton, loamy, sandy, and red chalaka. The population of the division is 2.57 million with a population density of 296 individuals per $\mathrm{km}^{2}$ (Anonymous 2011a). The area under forest cover is $2,310 \mathrm{~km}^{2}, 27 \%$ of the total geographical area of the division. The forest canopy density categories are moderately dense forest (953 $\mathrm{km}^{2}$ ), open forest (1015 km²), scrub $\left(91 \mathrm{~km}^{2}\right)$, and non-forest $\left(244 \mathrm{~km}^{2}\right)$. Water bodies occupy $6.7 \mathrm{~km}^{2}$ whereas reserved, protected, and un-classed forest categories cover $1,579 \mathrm{~km}^{2}, 709 \mathrm{~km}^{2}$, and $22 \mathrm{~km}^{2}$, respectively (Anonymous 2011b). The forest division has six ranges: Bhupalapally North, Eturnagaram, Tadvai, Pasra, Mulugu, and Warangal. The study site is in Tadvai and Eturnagaram ranges which include Eturnagaram Wildlife Sanctuary (Figure 1).

\section{Demography of study site}

The state of Andhra Pradesh, India, has $33(+2)$ scheduled tribes. Of these, Koyas are endemic to Godavari
Valley and are descendants of Gonds of central India (Sreeramulu 2009). They constitute a major endemic ethnic community whose medicinal plant knowledge is documented for intracultural study in the reserve forest area along the southern bank of the Godavari River, which lies between $18^{\circ} 10^{\prime}$ and $18^{\circ} 26^{\prime} \mathrm{N}$ and $80^{\circ} 09^{\prime}$ to $80^{\circ} 33^{\prime} \mathrm{E}$ (Figure 1). Land cover of the region supports two types of economic activity, namely seasonal agriculture (including podu [shifting] cultivation) and harvesting of forest produce (non-timber forest products).

\section{Research motivation}

An impetus to conduct ethnobotanical research in India was provided by the Ministry of Environment and Forests through the sanction of "All India Coordinated Research Project on Ethnobiology (AICRPE 1992-1998)." The project began with states starting with "A" such as Andhra Pradesh, Arunachal Pradesh, Andaman and Nicobar islands, etc. (Raju 2013). It paved the way for the first scientific and authentic work on ethnobotany of Eastern Ghats of Andhra Pradesh through the doctoral work by Rao (1988), whose findings were later published as a book (Rao \& Henry 1996). Rao (1988) visited Thupakulagudem village, which is part of Eturnagarm Wildlife Sanctuary, to document Koya ethnobotanical knowledge as part of intercultural study across the Eastern Ghats. Another intercultural study in the district was that of Reddy et al. (1998) on the ethnoveterinary phytomedicines. Later, Reddy et al. (2008) reported the phytotherapy practiced by Gonds, and Murthy et al. (2007) listed the plants used in ethnovet-

\section{District boundary}

\section{Eturnagaram Wildlife sanctuary}

Warangal North Division boundary

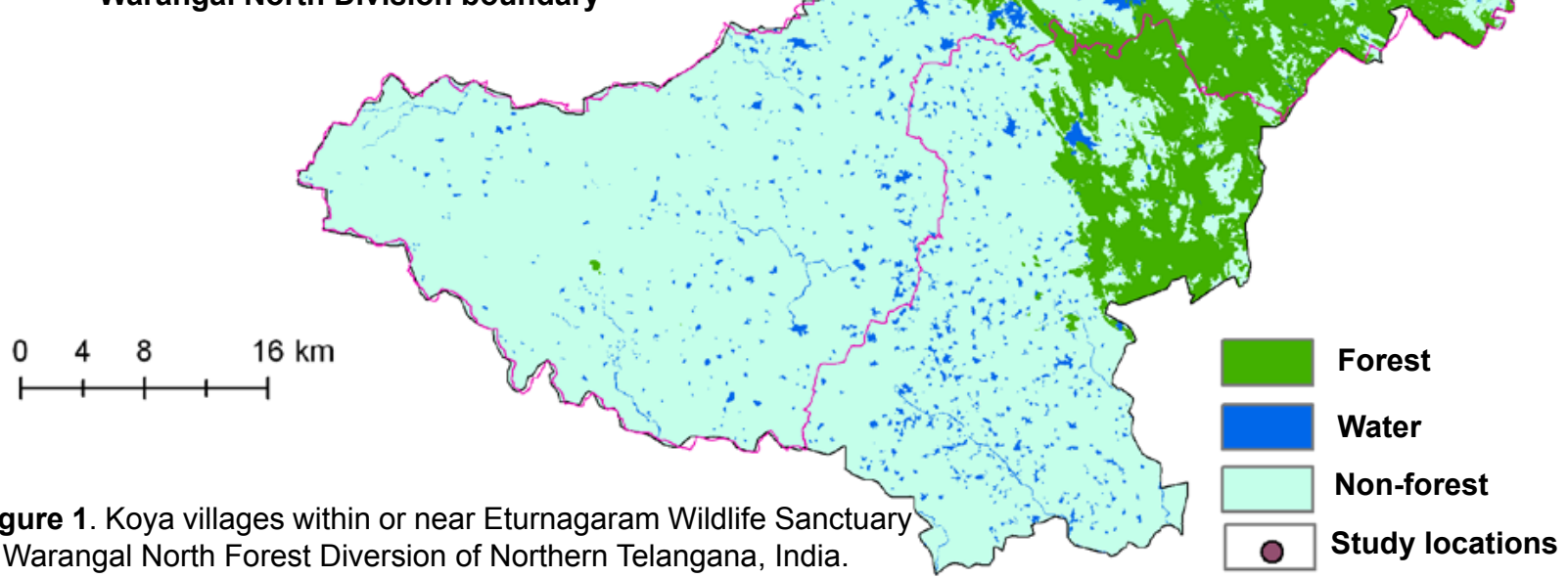

in Warangal North Forest Diversion of Northern Telangana, India.

Koya villages: A. Ailapur, B. Allamvari Ghanpur C. Banojibandam, D. Chalpaka, E. Chinnaboinapally, F. Dodla, G. Gandhinagar, H. Gonepally, I. Kamaram, J. Kondai, K. Mallur, L. Malyala, M. Medaram, N. Narlapur, O. Oorattum, P. Shapally, Q. Venkatapuram, and R. Yelishettipally. 


\section{Suthari et al. - Intracultural Cognizance of Medicinal Plants of Warangal North Forest Division, Northern Telangana, India}

erinary practices by Koyas of Pakhal Wildlife Sanctuary, which is part of the Warangal South Forest Division in the district. Recently, Sreeramulu (2009) compared the TBK of the local people of neighboring Nalgonda and Warangal districts in the region and demonstrated that those of the latter district are more knowledgeable of ethnobotanical plants. The present study adds to this body of knowledge by highlighting the medicinal plant usage of Koyas from villages near Eturnagaram Wildlife Sanctuary in Warangal North Forest Division.

\section{Methods}

\section{Ethnic villages}

Within the study area, 18 villages (Table 1) were chosen based on landscape heterogeneity, predominance of Koya inhabitants, and current use of local plants for medicinal purposes. The villagers are more or less equally impacted by village remoteness (proximity to the highway and developed towns with modern hospital facilities) and social and ecological changes. Mallur is one of two villages selected from outside the wildlife sanctuary to the

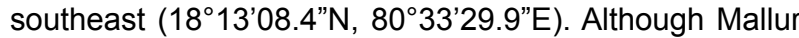
enjoys an almost similar physical environment, it is more culturally mixed (non-ethnic people) than other villages.
Moreover, Mallur village is also a medicinal plants conservation area (MPCA) and has a sacred grove (a non-ethnic [Hindu] temple) on a hill following the Chinthamani stream. Gandhinagar is the other control village located outside the wildlife sanctuary $\left(18^{\circ} 10^{\prime} 30.1^{\prime \prime} \mathrm{N}, 80^{\circ} 09^{\prime} 25.4 " \mathrm{E}\right)$ but is relatively far from the Godavari River in the southwest direction. An open degraded forest surrounds this village (Figure 1).

A premise of this study is that the ability to find and recognize medicinal plants is need-based and could be the same among villages but still vary with ethnic group and nature of forest habitat. The plants in a given habitat can be "apparent" (perennial and woody) or "non-apparent" (herbaceous which disappear at the end of the season; geophytes). The present study ensured that the sites (villages) compared are similar and there is minimal temporal difference in the data gathered. It is against this background that the levels of medical aid available and economic status of the ethnic peoples are the same for the villages. Therefore, it was presumed that the medicinal plant knowledge of the local people is homogeneous as well.

\section{Ethnic interviews and informants}

Ethnobotanical/ethnomedicinal data were collected through household surveys, questionnaires, and semi-

Table 1. Demographic information of Koya informants from 18 villages within or near Eturnagaram Wildlife Sanctuary in Warangal North Forest Division of Northern Telangana, India. Number of informants (\#). Gender: male (M), female (F).

\begin{tabular}{|c|c|c|c|c|c|c|c|}
\hline Village & Gender & \# & Age (yrs) & Village & Gender & $\#$ & Age (yrs) \\
\hline \multirow[t]{2}{*}{ Mallur } & $\mathrm{M}$ & 6 & $52,55,72,64,45,38$ & \multirow[t]{2}{*}{ Yelishettipally } & $\mathrm{M}$ & 5 & $50,53,57,41,44$ \\
\hline & $\mathrm{F}$ & 4 & $45,32,38,43$ & & $\mathrm{~F}$ & 3 & $37,46,57$ \\
\hline \multirow[t]{2}{*}{ Kamaram } & M & 4 & $63,59,41,48$ & \multirow[t]{2}{*}{ Banojibandam } & M & 2 & 44,53 \\
\hline & $\mathrm{F}$ & 3 & $41,37,44,55$ & & $\mathrm{~F}$ & 1 & 54 \\
\hline \multirow[t]{2}{*}{ Venkatapuram } & M & 4 & $52,59,64,51$ & \multirow[t]{2}{*}{ Chalpaka } & M & 5 & $51,57,59,65,48$ \\
\hline & $\mathrm{F}$ & 2 & 48,42 & & $\mathrm{~F}$ & 4 & $64,53,48,52$ \\
\hline \multirow[t]{2}{*}{ Chinnaboinapally } & M & 5 & $70,84,79,68,55$ & \multirow{2}{*}{$\begin{array}{l}\text { Allamvari } \\
\text { Ghanpur }\end{array}$} & M & 6 & $55,48,66,45,68,76$ \\
\hline & $\mathrm{F}$ & 2 & 53,57 & & $\mathrm{~F}$ & 2 & 58,63 \\
\hline \multirow[t]{2}{*}{ Shapally } & $M$ & 6 & $40,65,50,48,70,62$ & \multirow[t]{2}{*}{ Narlapur } & $M$ & 3 & $40,77,50$ \\
\hline & $\mathrm{F}$ & 2 & 55,48 & & $\mathrm{~F}$ & 4 & $46,38,65,45$ \\
\hline \multirow[t]{2}{*}{ Dodla } & M & 5 & $64,58,54,62,48$ & \multirow[t]{2}{*}{ Medaram } & M & 6 & $60,65,40,48,64,65$ \\
\hline & $\mathrm{F}$ & 3 & $35,42,57$ & & $\mathrm{~F}$ & 4 & $48,70,55,58$ \\
\hline \multirow[t]{2}{*}{ Malyala } & $\mathrm{M}$ & 7 & $\begin{array}{l}55,40,44,64,39,51, \\
56\end{array}$ & \multirow[t]{2}{*}{ Oorattum } & $\mathrm{M}$ & 3 & $68,46,55$ \\
\hline & $\mathrm{F}$ & 5 & $48,69,58,49,54$ & & $\mathrm{~F}$ & 2 & 42,49 \\
\hline \multirow[t]{2}{*}{ Kondai } & M & 8 & $\begin{array}{l}58,48,46,53,42,55 \\
66,63\end{array}$ & \multirow[t]{2}{*}{ Gonepally } & $M$ & 2 & 70,64 \\
\hline & $\mathrm{F}$ & 2 & 49,56 & & $\mathrm{~F}$ & 2 & 55,40 \\
\hline \multirow[t]{2}{*}{ Ailapur } & $M$ & 3 & $42,39,45$ & \multirow[t]{2}{*}{ Gandhinagar } & $\mathrm{M}$ & 2 & 68,55 \\
\hline & $\mathrm{F}$ & 3 & $48,54,52$ & & $\mathrm{~F}$ & 1 & 48 \\
\hline
\end{tabular}


structured and open-ended interviews. The interviews held for each village included a minimum of three informants (both genders) of the Koya community who were requested to gather in the village commons or one of their homes. The same questions were put to all informants selected for the study. Repeated (common or shared) uses and unique uses for plant species or disease categories were documented. Informants were shown plant specimens or pictures of species listed by other villages. To avoid variation in seasonal availability of medicinal plants, the study was conducted in the same season for all the sites. The 131 informants were all 32-84 years of age (Table 1). The criteria for informant selection were: (i) village residents for at least two decades, with a family hierarchy; (ii) podu cultivators or settled agriculturists; (iii) cattle owners; (iv) midwives; and (v) local healers (vaidyas or healthcare practitioners).

\section{Data collection and PIC}

The study of indigenous knowledge usually relies on interactions with a few members of the community to represent the knowledge held by the entire community. Many ethnobotanists adopt the cultural consensus model developed by Romney et al. (1986). Although this model has some limitations, it devalues differences in knowledge and focuses on consensus and was thus adopted for the present study.

Prior informed content (PIC) is an important concern underlying the philosophical and political environment of bioprospecting research involving indigenous societies (Rosenthal 2006). A scientific approach towards traditional botanical knowledge (TBK) holders began in Kakatiya University when the Government of India, through University Gants Commission, New Delhi, sanctioned a Special Assistance Program (SAP) in 2004. In compliance with the convention on biological diversity (CBD), PIC was established after relevant discussions between the university and the Koya community regarding the intended use of their knowledge. The mutual understanding was that any benefit derived from research pursued after their medicinal plant knowledge shall belong to them. Since TBK is a community knowledge and the purpose of the present survey is non-monetary, the Koya agreed to disclose information for the purposes of documenting their ability to recognize plant species by vernacular name and medicinal use. They readily consented because a prior survey in some of their villages helped to establish the Medicinal Plant Conservation Area (MPCA) at Mallur.

In India, there is a general and urgent need to pattern the process of PIC after educating the local ethnic groups in different regions of IPR, as per a standard scale. Furthermore, the majority of the local people in India are of the opinion that the knowledge could be displayed in full text for non-commercial and academic purposes (Singh 2008), as is the present case. However, something sophisticated that has happened on medicinal plant research in Guinea in regard to PIC and benefit-sharing in compliance with CBD (Carlson et al. 2001) should happen for India.

\section{Plant collections and identification}

Majority of plant species were identified either with the botanical name or local (vernacular/trivial) name by the authors in the field on the basis of their past taxonomic knowledge about these plants in the study area. The specimens of the unidentified species in the field were brought to the laboratory and identified with help of local floras (e.g. Gamble \& Fischer 1915-1935), eFloras (www. efloras.org), and recent taxonomic monographs. The currently accepted names of plant species are after www. ipni.org, www.plantlist.org, and www.tropicos.org. Voucher specimens were pressed, processed, and preserved at Kakatiya University Herbarium (KUW), Warangal.

\section{Data analysis}

The medicinal plant knowledge documented from Koyas across 18 villages was analyzed by plant species and family. The data were further classified as per the plant divisions and classes, growth forms (trees, shrubs, herbs, and climbers), ethnomedicine (human and veterinary), and nativity (indigenous and exotic). Analysis of variance was performed to determine presence of intervillage differences for the above categories.

The Family-Use Value (FUV) was calculated for top five families. It is based on sum of the species used within a given family ( $\Sigma U V s)$ in all 18 villages divided by the number of species that are used (ns) in the whole study area as per Hoffman \& Gallaher (2007).

\section{Results}

\section{Intracultural cognizance}

Overall the Koyas in the study area differed village-wise in both the number and kinds of species exploited. Analysis of variance showed significant intervillage differences relative to plant taxonomy (dicots versus monocots; $F=$ 30.247), growth form (trees, shrubs, herbs, climbers; $F=$ 11.496), medicinal target (humans, veterinary, both; $F=$ $25.827)$, and plant nativity $(F=23.835)$. All species reported by informants are listed in Table 2 along with their vernacular names, habit, parts used, and medicinal use.

Trees typically dominated the species list except in villages Chinnaboinapally and Allamvari Ghanpur where herbs are used more than the trees or climbers. These two villages are surrounded by secondary forests. The analysis of medicinal plant knowledge of Koyas in terms of growth forms revealed that $43 \%$ are trees, $30 \%$ herbs, $18 \%$ climbers, and $9 \%$ shrubs (Table 3 ). Patterns of growth form use are indicative of certain trends: (i) local people still primar- 


\section{Suthari et al. - Intracultural Cognizance of Medicinal Plants of Warangal North Forest Division, Northern Telangana, India}

Table 2. Names, families, habit, parts used, and ethnomedicinal use of plant species reported by Koya informants from 18 villages within or near Eturnagaram Wildlife Sanctuary in Warangal North Forest Division of Northern Telangana, India. Habit: climber (C), epiphyte $(E)$, fern $(F)$, herb $(H)$, shrub $(S)$, straggler $(S t)$, tree (T), twiner (Tw). Parts used: bark $(B)$, flower $(F I)$, fruit $(F r)$, gum $(G)$, latex $(\mathrm{La})$, leaf $(\mathrm{L})$, root $(R)$, seed $(\mathrm{Se})$, tender sticks $(\mathrm{TS})$, tuber $(\mathrm{T})$, whole plant (W).

\begin{tabular}{|c|c|c|c|c|c|}
\hline \multicolumn{2}{|l|}{ Botanical Names } & \multirow{2}{*}{$\begin{array}{l}\text { Voucher } \\
\text { Number }\end{array}$} & \multirow[t]{2}{*}{ Habit } & \multirow{2}{*}{$\begin{array}{l}\text { Part(s) } \\
\text { used }\end{array}$} & \multirow[t]{2}{*}{ Ethnic use } \\
\hline Scientific & Vernacular & & & & \\
\hline \multicolumn{6}{|l|}{ ACANTHACEAE } \\
\hline $\begin{array}{l}\text { Andrographis paniculata (Burm.f.) } \\
\text { Wall. ex Nees }\end{array}$ & Nelavemu & 307 & $\mathrm{H}$ & W & Dyspepsia \\
\hline Barleria cristata L. & Neeli gorinta & 643 & $\mathrm{H}$ & $R$ & Abortifacient \\
\hline Barleria montana Nees & Nilambaram & 881 & $S$ & $R$ & Skin disease \\
\hline Barleria prionitis L. & Mulla gorinta & 201 & $\mathrm{H}$ & $R$ & Scorpion bite \\
\hline Hemigraphis latebrosa (Roth) Nees & Akkala & 819 & $\mathrm{H}$ & $R$ & Anti-alcoholic agent \\
\hline $\begin{array}{l}\text { Hygrophila auriculata (Schumach.) } \\
\text { Heine }\end{array}$ & Gorimidi & 533 & $\mathrm{H}$ & $L$ & Dropsy \\
\hline Justicia betonica L. & \begin{tabular}{|l|} 
Pedda \\
nilambaram
\end{tabular} & 119 & $S$ & $R$ & Mental disorders \\
\hline Lepidagathis cristata Willd. & $\begin{array}{l}\text { Nakka } \\
\text { peetirigadda }\end{array}$ & 644 & $\mathrm{H}$ & $\mathrm{R} / \mathrm{W}$ & Fever, fits \\
\hline $\begin{array}{l}\text { Peristrophe paniculata (Forssk.) } \\
\text { Brummitt }\end{array}$ & Chebura & 287 & $\mathrm{H}$ & $L$ & Skin disease \\
\hline \multicolumn{6}{|l|}{ AMARANTHACEAE } \\
\hline Achyranthes aspera L. & Uttareni & 63 & $\mathrm{H}$ & $R$ & Scorpion bite \\
\hline Aerva lanata (L.) Juss. & Pindi kura & 19 & $\mathrm{H}$ & $L$ & Wounds \\
\hline Aerva sanguinolenta (L.) Blume & Konda pindi & 1400 & $\mathrm{H}$ & W & Urinary troubles \\
\hline Amaranthus spinosus L. & \begin{tabular}{|l|} 
Mulla \\
thotakura
\end{tabular} & 280 & $\mathrm{H}$ & $L$ & Appetite stimulant \\
\hline Celosia argentea L. & Gunugu & 64 & $\mathrm{H}$ & $L$ & Cuts \\
\hline Pupalia lappacea (L.) Juss. & $\begin{array}{l}\text { Gundu } \\
\text { uttareni }\end{array}$ & 620 & $\mathrm{H}$ & $L$ & Insect bite \\
\hline \multicolumn{6}{|l|}{ ANACARDIACEAE } \\
\hline $\begin{array}{l}\text { Buchanania axillaris (Desr.) } \\
\text { Ramamoorthy }\end{array}$ & Pedda morli & 329 & $\mathrm{~T}$ & $B / G$ & $\begin{array}{l}\text { Veterinary, skin } \\
\text { disease, back pain }\end{array}$ \\
\hline $\begin{array}{l}\text { Buchanania cochinchinensis (Lour.) } \\
\text { M.R.Almeida }\end{array}$ & Morri, Morli & 259 & $\mathrm{~T}$ & G & Back pain, laxative \\
\hline Lannea coromandelica (Houtt.) Merr. & $\begin{array}{l}\text { Dumpena, } \\
\text { Dumpidi } \\
\end{array}$ & 863 & $\mathrm{~T}$ & $B$ & $\begin{array}{l}\text { Antiseptic, appetite } \\
\text { stimulant }\end{array}$ \\
\hline Mangifera indica L. & Konda mamidi & 1012 & $\mathrm{~T}$ & $\mathrm{~B} / \mathrm{Fr}$ & Appetite stimulant, tonic \\
\hline Semecarpus anacardium L.f. & Nalla jeedi & 261 & $\mathrm{~T}$ & $\mathrm{Se}$ & Wounds \\
\hline \multicolumn{6}{|l|}{ ANNONACEAE } \\
\hline Miliusa tomentosa (Roxb.) J.Sinclair & Barre duddi & 853 & $\mathrm{~T}$ & B & Veterinary \\
\hline Polyalthia cerasoides (Roxb.) Bedd. & Chiluka duddi & 321 & $\mathrm{~T}$ & B & Veterinary \\
\hline \multicolumn{6}{|l|}{ APOCYNACEAE } \\
\hline Anodendron paniculatum A.DC. & $\begin{array}{l}\text { Athukudu } \\
\text { teega }\end{array}$ & 1631 & $\mathrm{C}$ & $B$ & Bone fracture \\
\hline Calotropis gigantea (L.) Dryand. & Jilledu & 12 & $S$ & $R$ & Snake bite \\
\hline
\end{tabular}




\begin{tabular}{|c|c|c|c|c|c|}
\hline \multicolumn{2}{|l|}{ Botanical Names } & \multirow{2}{*}{$\begin{array}{l}\text { Voucher } \\
\text { Number }\end{array}$} & \multirow[t]{2}{*}{ Habit } & \multirow{2}{*}{$\begin{array}{l}\text { Part(s) } \\
\text { used }\end{array}$} & \multirow[t]{2}{*}{ Ethnic use } \\
\hline Scientific & Vernacular & & & & \\
\hline $\begin{array}{l}\text { Cyptolepis dubia (Burm.f.) } \\
\text { M.R.Almeida }\end{array}$ & Budda pala & 304 & $\mathrm{C}$ & $R$ & Veterinary \\
\hline $\begin{array}{l}\text { Hemidesmus indicus (L.) R.Br. } \\
\text { ex Schult. }\end{array}$ & Sugandhi pala & 331 & C & $\mathrm{R}$ & Blood purifier, sugar control \\
\hline $\begin{array}{l}\text { Hemidesmus indicus var. pubescens } \\
\text { (Wight \& Arn.) Hook.f. }\end{array}$ & \begin{tabular}{|l|} 
Barre \\
sugandhi pala
\end{tabular} & 55 & C & $\mathrm{R}$ & Diabetes \\
\hline Holarrhena pubescens Wall ex G.Don & Istari pala & 269 & $\mathrm{~T}$ & $\mathrm{~B}$ & Dysentery \\
\hline Pergularia daemia (Forssk.) Chiov. & Dustapu teega & 121 & $\mathrm{C}$ & W & Respiratory disease \\
\hline \multicolumn{6}{|l|}{ ARACEAE } \\
\hline Pistia stratiotes L. & $\begin{array}{l}\text { Antara } \\
\text { thamara }\end{array}$ & 558 & $\mathrm{H}$ & W & Baldness \\
\hline \multicolumn{6}{|l|}{ ARECACEAE } \\
\hline Phoenix loureiroi Kunth & Jittetha & 1650 & $\mathrm{~T}$ & $\mathrm{Fr}$ & Laxative \\
\hline \multicolumn{6}{|l|}{ ARISTOLOCHIACEAE } \\
\hline Aristolochia indica L. & Nalleswari & 145 & $\mathrm{C}$ & $\mathrm{R}$ & Snake bite \\
\hline \multicolumn{6}{|l|}{ ASPARAGACEAE } \\
\hline Asparagus gonoclados Baker & $\begin{array}{l}\text { Guddelugu } \\
\text { bochu }\end{array}$ & 316 & C & $T$ & Aphrodisiac \\
\hline Asparagus racemosus Willd. & Pilli teegalu & 1624 & $\mathrm{C}$ & $T$ & Aphrodisiac \\
\hline \multicolumn{6}{|l|}{ ASTERACEAE } \\
\hline Blumea bifoliata (L.) DC. & Kukka pogaku & 968 & $\mathrm{H}$ & $\mathrm{R}$ & Cough \\
\hline Blumea virens DC. & Adavi pogaku & 945 & $\mathrm{H}$ & $\mathrm{L}$ & Skin disease \\
\hline Pentanema indicum (L.) Ling & $\begin{array}{l}\begin{array}{l}\text { Adavi } \\
\text { chamanti }\end{array} \\
\end{array}$ & 815 & $\mathrm{H}$ & $\mathrm{R}$ & Abortifacient \\
\hline Sphaeranthus indicus L. & Bodasaram & 50 & $\mathrm{H}$ & W & Kill lice in domestic fowl \\
\hline Tridax procumbens (L.) L. & Nalla alam & 31 & $\mathrm{H}$ & $\mathrm{L}$ & Hemorrhage, cuts \\
\hline Vernonia cinerea (L.) Less. & Garita kamma & 80 & $\mathrm{H}$ & $\mathrm{R}$ & Fever \\
\hline $\begin{array}{l}\text { Vernonia cinerea var. parviflora } \\
\text { (Reinw. ex Blume) DC. }\end{array}$ & $\begin{array}{l}\text { Adavi garita } \\
\text { kamma }\end{array}$ & 1652 & $\mathrm{H}$ & $\mathrm{R}$ & Fever \\
\hline \multicolumn{6}{|l|}{ BIGNONIACEAE } \\
\hline Millingtonia hortensis L.f. & Boda malle & 125 & $\mathrm{~T}$ & $\mathrm{~B}$ & Bronchitis \\
\hline Oroxylum indicum (L.) Kurz & Dundilam & 708 & $\mathrm{~T}$ & $\mathrm{~B}$ & Rheumatism \\
\hline Stereospermum chelonoides (L.f.) DC. & Kalagoru & 597 & $\mathrm{~T}$ & $\mathrm{~B}$ & Veterinary \\
\hline \multicolumn{6}{|l|}{ BOMBACACEAE } \\
\hline Bombax ceiba L. & Buruga & 213 & $\mathrm{~T}$ & $\mathrm{~B}$ & Cough \\
\hline \multicolumn{6}{|l|}{ BORAGINACEAE } \\
\hline Coldenia procumbens $\mathrm{L}$. & $\begin{array}{l}\text { Cheppu } \\
\text { tattaku }\end{array}$ & 200 & $\mathrm{H}$ & W & Wounds \\
\hline Cordia dichotoma G.Forst. & Iriki & 640 & $\mathrm{~T}$ & $\mathrm{Fr}$ & Dyspepsia \\
\hline Cordia macleodii Hook.f. \& Thomson & Botuku & 704 & $\mathrm{~T}$ & $\mathrm{~B}$ & Veterinary \\
\hline Ehretia laevis Roxb. & Pala danthi & 888 & $\mathrm{~T}$ & $\mathrm{Fr}$ & Dyspepsia \\
\hline Trichodesma indicum (L.) Lehm. & Gabba & 56 & $\mathrm{H}$ & $\mathrm{L}$ & Wounds \\
\hline BURSERACEAE & & & & & \\
\hline
\end{tabular}




\section{Suthari et al. - Intracultural Cognizance of Medicinal Plants of Warangal North Forest Division, Northern Telangana, India}

\begin{tabular}{|c|c|c|c|c|c|}
\hline \multicolumn{2}{|l|}{ Botanical Names } & \multirow{2}{*}{$\begin{array}{l}\text { Voucher } \\
\text { Number }\end{array}$} & \multirow[t]{2}{*}{ Habit } & \multirow{2}{*}{$\begin{array}{l}\text { Part(s) } \\
\text { used }\end{array}$} & \multirow[t]{2}{*}{ Ethnic use } \\
\hline Scientific & Vernacular & & & & \\
\hline Boswellia serrata Roxb. ex Colebr. & Anduga & 354 & $T$ & B & Fever, tonic \\
\hline Garuga pinnata Roxb. & Garugu & 978 & $T$ & $\mathrm{~B}$ & Veterinary \\
\hline \multicolumn{6}{|l|}{ CAPPARACEAE } \\
\hline Capparis sepiaria L. & Uppi & 941 & St & B & Veterinary \\
\hline Capparis zeylanica L. & Aadonda & 373 & C & $\mathrm{R} / \mathrm{Fr}$ & Dyspepsia, tonic \\
\hline \multicolumn{6}{|l|}{ CARYOPHYLLACEAE } \\
\hline Polycarpaea corymbosa (L.) Lam. & Gaddi puvvu & 116 & $\mathrm{H}$ & $\mathrm{R}$ & Snake bite \\
\hline \multicolumn{6}{|l|}{ CELASTRACEAE } \\
\hline Cassine glauca (Rottb.) Kuntze & Bhutankush & 606 & $T$ & $\mathrm{~B}$ & Dyspepsia \\
\hline $\begin{array}{l}\text { Gymnosporia emarginata (Willd.) } \\
\text { Thwaites }\end{array}$ & Danthi & 257 & $T$ & B & Skin disease \\
\hline \multicolumn{6}{|l|}{ CLEOMACEAE } \\
\hline Cleome viscosa L. & Vaminta & 2 & $\mathrm{H}$ & $\mathrm{W}$ & Skin disease \\
\hline \multicolumn{6}{|l|}{ COMBRETACEAE } \\
\hline $\begin{array}{l}\text { Anogeissus acuminata (Roxb. ex DC.) } \\
\text { Wall. ex Guillem. \& Perr. }\end{array}$ & Pasi & 1639 & $\mathrm{~T}$ & L & Cough \\
\hline Combretum albidum G.Don & Yada teega & 338 & $\mathrm{C}$ & $B$ & Veterinary \\
\hline Combretum roxburghii Spreng. & Yadaku & 878 & $\mathrm{C}$ & $\mathrm{B}$ & Veterinary \\
\hline Combretum latifolium Blume & Yada teega & 1547 & $\mathrm{C}$ & B & Veterinary \\
\hline Getonia floribunda Roxb. & Bontha & 264 & C & $B$ & Veterinary \\
\hline $\begin{array}{l}\text { Terminalia arjuna (Roxb. ex DC.) } \\
\text { Wight \& Arn. }\end{array}$ & $\begin{array}{l}\text { Yeru maddi, } \\
\text { Tella maddi }\end{array}$ & 337 & $T$ & $B$ & Wounds, heart disease \\
\hline Terminalia bellirica (Gaertn.) Roxb. & Tani & 879 & $T$ & $\mathrm{Fr}$ & Cough \\
\hline Terminalia chebula Retz. & Karakkaya & 978 & $T$ & $\mathrm{Fr}$ & Cough \\
\hline \multicolumn{6}{|l|}{ CONVOLVULACEAE } \\
\hline $\begin{array}{l}\text { Ipomoea coptica (L.) Roth ex Roem. } \\
\text { \& Schult. }\end{array}$ & Suvarchala & 900 & C & L & Mouth wash \\
\hline Ipomoea eriocarpa R.Br. & Elika chevi & 397 & C & $\mathrm{R}$ & Stomach-ache \\
\hline Ipomoea hederifolia L. & Kashiratnam & 594 & C & $\mathrm{R}$ & Tonic \\
\hline Ipomoea obscura (L.) Ker Gawl. & Macha aku & 453 & C & $\mathrm{L}$ & Insect bite \\
\hline $\begin{array}{l}\text { Merremia hederacea (Burm.f.) } \\
\text { Hallier f. }\end{array}$ & Talantu teega & 885 & C & $\mathrm{Fr}$ & Hair wash \\
\hline Rivea hypocrateriformis Choisy & Boddi kura & 596 & C & $\mathrm{R}$ & Snake bite \\
\hline \multicolumn{6}{|l|}{ CORNACEAE } \\
\hline Alangium salviifolium (L.f.) Wangerin. & Ooduga & 1004 & $\mathrm{~T}$ & B & Snake bite \\
\hline \multicolumn{6}{|l|}{ CUCURBITACEAE } \\
\hline Diplocyclos palmatus (L.) C.Jeffrey & Linga donda & 84 & C & $\mathrm{Fr}$ & Diarrhea \\
\hline Momordica charantia L. & Kakara & 158 & C & $\mathrm{Fr}$ & Diabetes \\
\hline Momordica dioica Roxb. ex Willd. & Boda kakara & 579 & C & $\mathrm{Fr}$ & Diabetes \\
\hline \multicolumn{6}{|l|}{ CYPERACEAE } \\
\hline Cyperus rotundus L. & Thunga & 27 & $\mathrm{H}$ & $T / R$ & Tonic, scorpion bite \\
\hline Scleria levis Retz. & Ashta medha & 1316 & $\mathrm{H}$ & $\mathrm{R}$ & Blood purifier \\
\hline
\end{tabular}




\begin{tabular}{|c|c|c|c|c|c|}
\hline \multicolumn{2}{|l|}{ Botanical Names } & \multirow{2}{*}{$\begin{array}{l}\text { Voucher } \\
\text { Number }\end{array}$} & \multirow[t]{2}{*}{ Habit } & \multirow{2}{*}{$\begin{array}{l}\text { Part(s) } \\
\text { used }\end{array}$} & \multirow[t]{2}{*}{ Ethnic use } \\
\hline Scientific & Vernacular & & & & \\
\hline \multicolumn{6}{|l|}{ DILLENIACEAE } \\
\hline Dillenia pentagyna Roxb. & Kalinga & 1028 & $\mathrm{~T}$ & $\mathrm{~B} / \mathrm{Fr}$ & Tonic, fever, veterinary \\
\hline \multicolumn{6}{|l|}{ DIOSCOREACEAE } \\
\hline Dioscorea bulbifera L. & Chenna gadda & 1100 & $\mathrm{C}$ & $\mathrm{T}$ & Arthritis, fever \\
\hline \multicolumn{6}{|l|}{ EBENACEAE } \\
\hline Diospyros chloroxylon Roxb. & Illinda & 616 & $\mathrm{~T}$ & $B$ & Veterinary \\
\hline Diospyros malabarica (Desr.) Kostel. & Konda tuniki & 54 & $\mathrm{~T}$ & B & Bronchitis \\
\hline Diospyros melanoxylon Roxb. & Tuniki & 335 & $\mathrm{~T}$ & $\mathrm{Fr}$ & Dyspepsia, laxative \\
\hline Diospyros montana Roxb. & Muchi tuniki & 894 & $\mathrm{~T}$ & $\mathrm{~B} / \mathrm{Fr}$ & Veterinary \\
\hline \multicolumn{6}{|l|}{ ERYTHROXYLACEAE } \\
\hline Erythroxylum monogynum Roxb. & Deva daru & 1029 & $\mathrm{~T}$ & $\mathrm{Fr}$ & Tonic \\
\hline \multicolumn{6}{|l|}{ EUPHORBIACEAE } \\
\hline Acalypha indica L. & Penta puti & 22 & $\mathrm{H}$ & $\mathrm{W}$ & Skin disease \\
\hline Euphorbia hirta L. & \begin{tabular}{|l} 
Reddivari \\
nanubalu \\
\end{tabular} & 23 & $\mathrm{H}$ & L & Wounds \\
\hline Euphorbia nivulia Buch.-Ham. & Aku jemudu & 1640 & $\mathrm{~T}$ & $\mathrm{La}$ & Bone fracture \\
\hline Mallotus philippensis (Lam.) Müll.Arg. & Kunkuma & 1700 & $\mathrm{~T}$ & $\mathrm{Se}$ & Leucoderma \\
\hline Microstachys chamaelea (L.) Müll.Arg. & Isaka & 954 & $\mathrm{H}$ & $\mathrm{R}$ & Skin disease \\
\hline Tragia involucrata L. & Dula gondi & 953 & $\mathrm{C}$ & $\mathrm{R}$ & Scorpion bite \\
\hline \multicolumn{6}{|l|}{ FABACEAE } \\
\hline Abrus precatorius $\mathrm{L}$. & Gurija & 5 & $\mathrm{C}$ & $\mathrm{Se}$ & Abortifacient \\
\hline Acacia chundra (Rottler) Willd. & Sandra & 613 & $\mathrm{~T}$ & $\mathrm{~B}$ & Appetite stimulant \\
\hline Acacia nilotica (L.) Willd. ex Del. & Nalla tumma & 236 & $\mathrm{~T}$ & $B$ & Burns \\
\hline Albizia odoratissima (L.f.) Benth. & Chinduga & 731 & $\mathrm{~T}$ & $B$ & Leucoderma \\
\hline Bauhinia malabarica Roxb. & Puli are & 1651 & $\mathrm{~T}$ & $\mathrm{~B}$ & Dyspepsia \\
\hline Bauhinia racemosa Lam. & Are & 87 & $\mathrm{~T}$ & B & Leucoderma \\
\hline Bauhinia semla Wunderlin & Goddeti are & 1490 & $\mathrm{~T}$ & $B$ & Tonic \\
\hline Butea superba Roxb. & Teega moduga & 328 & $\mathrm{C}$ & $\mathrm{B}$ & Urinary troubles \\
\hline Canavalia cathartica Thouars & Adavi tamba & 732 & $\mathrm{C}$ & $\mathrm{Se}$ & Scorpion bite \\
\hline Cassia fistula L. & Rela & 132 & $\mathrm{~T}$ & $\mathrm{Fr}$ & Diarrhea, diuretic \\
\hline Crotalaria albida B.Heyne ex Roth & Adavi janumu & 1222 & $\mathrm{H}$ & $\mathrm{R}$ & Scorpion bite \\
\hline Crotalaria ramosissima Roxb. & Tella janumu & 865 & $\mathrm{H}$ & $\mathrm{L}$ & Wounds \\
\hline Crotalaria verrucosa L. & Tella janumu & 41 & $\mathrm{H}$ & $\mathrm{R}$ & Snake bite \\
\hline $\begin{array}{l}\text { Dalbergia lanceolaria subsp. } \\
\text { paniculata (Roxb.) Thoth. }\end{array}$ & Porla pachari & 359 & $\mathrm{~T}$ & B & Baldness \\
\hline Dalbergia volubilis Roxb. & Teega pachari & 1340 & $\mathrm{C}$ & $\mathrm{B}$ & Skin disease \\
\hline Derris scandens (Roxb.) Benth. & Chakali teega & 346 & $\mathrm{C}$ & $\mathrm{B}$ & Veterinary \\
\hline Desmodium gangeticum (L.) DC. & Konda saru & 260 & $\mathrm{H}$ & $\mathrm{R}$ & Scorpion bite \\
\hline Desmodium triflorum (L.) DC. & Munta mandu & 378 & $\mathrm{~T}$ & $\mathrm{R}$ & Hemorrhage \\
\hline Desmodium velutinum (Willd.) DC. & Teega velga & 654 & $\mathrm{H}$ & $\mathrm{R}$ & Scorpion bite \\
\hline
\end{tabular}




\section{Suthari et al. - Intracultural Cognizance of Medicinal Plants of Warangal North Forest Division, Northern Telangana, India}

\begin{tabular}{|c|c|c|c|c|c|}
\hline \multicolumn{2}{|l|}{ Botanical Names } & \multirow{2}{*}{$\begin{array}{l}\text { Voucher } \\
\text { Number }\end{array}$} & \multirow[t]{2}{*}{ Habit } & \multirow{2}{*}{$\begin{array}{l}\text { Part(s) } \\
\text { used }\end{array}$} & \multirow[t]{2}{*}{ Ethnic use } \\
\hline Scientific & Vernacular & & & & \\
\hline $\begin{array}{l}\text { Dichrostachys cinerea (L.) Wight } \\
\text { \& Arn. }\end{array}$ & Veluthuru & 582 & $s$ & $B$ & Skin disease \\
\hline Erythrina suberosa Roxb. & Muli moduga & 1405 & $T$ & $B$ & \begin{tabular}{|l|} 
Veterinary, appetite \\
stimulant
\end{tabular} \\
\hline Flemingia macrophylla (Willd.) Merr. & Err puvvu & 1625 & $\mathrm{~s}$ & $\mathrm{R}$ & Skin disease \\
\hline Flemingia strobilifera (L.) W.T.Aiton & Adavi chappa & 1339 & $\mathrm{~S}$ & $\mathrm{R}$ & Skin disease \\
\hline Hardwickia binata Roxb. & Narepa & 1031 & $\mathrm{~T}$ & $B$ & Veterinary \\
\hline Indigofera cassioides Rottler ex DC. & Karu kandi & 609 & $S$ & $\mathrm{R}$ & Scorpion bite \\
\hline Mimosa hamata Willd. & Magadadi & 615 & $S$ & $B$ & Aphrodisiac \\
\hline Mucuna pruriens (L.) DC. & Dula gondi & 868 & $\mathrm{C}$ & $\mathrm{Se}$ & Abortifacient, aphrodisiac \\
\hline Paracalyx scariosus (Roxb.) Ali & $\begin{array}{l}\text { Adavi tella } \\
\text { kandi }\end{array}$ & 1635 & C & $R$ & Skin disease \\
\hline Phyllodium pulchellum (L.) Desv. & Sarivi & 1381 & $\mathrm{H}$ & $\mathrm{R}$ & Scorpion bite \\
\hline Pongamia pinnata (L.) Pierre & Kanuga & 133 & $\mathrm{~T}$ & $B$ & Skin disease \\
\hline Pueraria tuberosa (Willd.) DC. & Nela gummadi & 567 & $\mathrm{H}$ & $\mathrm{T}$ & All diseases \\
\hline Rhynchosia suaveolens (L.f.) DC. & Adavi kandi & 1098 & $S$ & $\mathrm{Se}$ & Dysentery \\
\hline Senna alata (L.) Roxb. & & 715 & $\mathrm{H}$ & $\mathrm{L}$ & Skin disease \\
\hline Senna occidentalis (L.) Link & $\begin{array}{l}\text { Adavi } \\
\text { chennangi }\end{array}$ & 149 & $\mathrm{H}$ & $\mathrm{Fr}$ & Appetite stimulant \\
\hline Senna tora (L.) Roxb. & Tagarisa & 8 & $\mathrm{H}$ & $\mathrm{L}$ & Insect bite \\
\hline Sophora velutina Lindl. & Adavi kanuga & 870 & $\mathrm{~T}$ & $B$ & Appetite stimulant \\
\hline Tamarindus indica L. & Chinta & 156 & $\mathrm{~T}$ & $\mathrm{~B}$ & Scorpion bite \\
\hline Tephrosia purpurea (L.) Pers. & Vempali & 6 & $\mathrm{H}$ & $\mathrm{R}$ & Easy delivery \\
\hline Xylia xylocarpa (Roxb.) Taub. & Bojja & 273 & $\mathrm{~T}$ & B & Skin disease \\
\hline \multicolumn{6}{|l|}{ FERN AND FERN ALLIES } \\
\hline Ceratopteris thalictroides (L.) Brongn. & Medha & 1213 & $F / S$ & $W$ & Memory \\
\hline Cyclosorus parasiticus (L.) Farw. & Hamsa padu & 655 & $\mathrm{~F} / \mathrm{H}$ & $W$ & Mental disorders \\
\hline Lygodium flexuosum (L.) Sw. & Dayyapu jeda & 982 & $F / C$ & $\mathrm{R}$ & Skin disease \\
\hline Selaginella bryopteris (L.) Baker & Pittakalu & 1653 & $\mathrm{H}$ & W & Memory \\
\hline \multicolumn{6}{|l|}{ GISEKIACEAE } \\
\hline Gisekia pharnaceoides L. & Iska rasi & 1427 & $\mathrm{H}$ & $\mathrm{W}$ & Pains \\
\hline \multicolumn{6}{|l|}{ HYPOXIDACEAE } \\
\hline Curculigo orchioides Gaertn. & Nela thadi & 603 & $\mathrm{H}$ & $\mathrm{T}$ & Aphrodisiac \\
\hline \multicolumn{6}{|l|}{ LAMIACEAE } \\
\hline $\begin{array}{l}\text { Anisomeles malabarica (L.) R.Br. } \\
\text { ex Sims }\end{array}$ & Dayyam chettu & 646 & $\mathrm{H}$ & W & Fever \\
\hline Gmelina arborea Roxb. & Gummadi teku & 354 & $T$ & $B$ & $\begin{array}{l}\text { Anti-inflammatory, } \\
\text { veterinary }\end{array}$ \\
\hline Leucas aspera (Willd.) Link & Thummi & 72 & $\mathrm{H}$ & $\mathrm{L}$ & Healing power \\
\hline Leucas decemdentata (Willd.) Sm. & Konda tummi & 1213 & $\mathrm{H}$ & $\mathrm{L}$ & Skin disease \\
\hline Ocimum americanum $\mathrm{L}$. & Kukka tulasi & 17 & $\mathrm{H}$ & $\mathrm{L}$ & Antiseptic \\
\hline Plectranthus mollis (Aiton) Spreng. & Nela marri & 600 & $\mathrm{H}$ & $\mathrm{R}$ & Scorpion bite \\
\hline
\end{tabular}




\begin{tabular}{|c|c|c|c|c|c|}
\hline \multicolumn{2}{|l|}{ Botanical Names } & \multirow{2}{*}{$\begin{array}{l}\text { Voucher } \\
\text { Number }\end{array}$} & \multirow[t]{2}{*}{ Habit } & \multirow{2}{*}{$\begin{array}{l}\text { Part(s) } \\
\text { used }\end{array}$} & \multirow[t]{2}{*}{ Ethnic use } \\
\hline Scientific & Vernacular & & & & \\
\hline Symphorema involucratum Roxb. & Konda takkali & 697 & C & $B$ & Veterinary \\
\hline Tectona grandis L.f. & Teku & 308 & $\mathrm{~T}$ & $B$ & Abortifacient \\
\hline \multicolumn{6}{|l|}{ LAURACEAE } \\
\hline Litsea glutinosa (Lour.) C.B. Rob. & Narra mamidi & 567 & $\mathrm{~T}$ & $B$ & All diseases \\
\hline \multicolumn{6}{|l|}{ LECYTHIDACEAE } \\
\hline Barringtonia acutangula (L.) Gaertn. & Nir kanki & 301 & $\mathrm{~T}$ & $B$ & Veterinary \\
\hline Careya arborea Roxb. & Budadharmi & 1360 & $\mathrm{~T}$ & $\mathrm{Fr} / \mathrm{B}$ & Cough, appetite stimulant \\
\hline \multicolumn{6}{|l|}{ LINDERNIACEAE } \\
\hline Lindernia ciliata (Colsm.) Pennell & Nela rampi & 1108 & $\mathrm{H}$ & W & Skin disease \\
\hline \multicolumn{6}{|l|}{ LOGANIACEAE } \\
\hline Strychnos nux-vomica L. & Visha mushti & 270 & $\mathrm{~T}$ & B & Snake bite, antiseptic \\
\hline Strychnos potatorum L.f. & Chilla & 332 & $\mathrm{~T}$ & $\mathrm{Se}$ & Eye disease \\
\hline \multicolumn{6}{|l|}{ LORANTHACEAE } \\
\hline Dendrophthoe falcata (L.f.) Ettingsh. & Vadanica & 850 & $\mathrm{H}$ & W & Fever \\
\hline \multicolumn{6}{|l|}{ LYTHRACEAE } \\
\hline Lagerstroemia parviflora Roxb. & Chennangi & 265 & $\mathrm{~T}$ & B & Veterinary \\
\hline Woodfordia fruticosa (L.) Kurz & Jaji & 1033 & $\mathrm{~T}$ & $B$ & Tonic \\
\hline \multicolumn{6}{|l|}{ MALVACEAE } \\
\hline Abutilon indicum (L.) Sweet & Mudra benda & 3 & $\mathrm{H}$ & $R$ & Aphrodisiac \\
\hline Eriolaena hookeriana Wight \& Arn. & Bothuku & 607 & $\mathrm{~T}$ & B & Veterinary \\
\hline Grewia hirsuta Vahl & Jibilika & 350 & $\mathrm{~s}$ & $\mathrm{Fr}$ & Laxative \\
\hline Grewia damine Gaertn. & Adavi jana & 608 & $\mathrm{~s}$ & $\mathrm{Fr}$ & Laxative \\
\hline Grewia rothii DC. & $\begin{array}{l}\text { Jana, Chinna } \\
\text { jana }\end{array}$ & 836 & 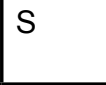 & $\mathrm{Fr}$ & Laxative \\
\hline Grewia tiliifolia Vahl & Tada & 326 & $\mathrm{~s}$ & $\mathrm{~B}$ & Veterinary \\
\hline Helicteres isora L. & Nulthada & 276 & $S$ & B & Veterinary \\
\hline Hibiscus panduriformis Burm.f. & Adavi benda & 842 & $S$ & $\mathrm{R}$ & Skin disease \\
\hline Kavalama urens (Roxb.) Raf. & Tabsi & 322 & $\mathrm{~T}$ & $B$ & Appetite stimulant \\
\hline Kydia calycina Roxb. & Konda patti & 1234 & $\mathrm{~T}$ & $\mathrm{~B}$ & Dyspepsia \\
\hline $\begin{array}{l}\text { Pterospermum xylocarpum (Gaertn.) } \\
\text { Santapau \& Wagh }\end{array}$ & Lolugu & 1591 & $\mathrm{~T}$ & $B$ & Veterinary \\
\hline Sida acuta Burm.f. & Chilka parre & 34 & $\mathrm{H}$ & $\mathrm{R}$ & Mental disorders \\
\hline Sida cordata (Burm.f.) Borss. Waalk. & Gaya paku & 253 & $\mathrm{H}$ & $R$ & Tonic \\
\hline Sida cordifolia L. & Chiru benda & 401 & $\mathrm{H}$ & $\mathrm{R}$ & Appetite stimulant \\
\hline $\begin{array}{l}\text { Thespesia lampas (Cav.) Dalzell \& } \\
\text { A.Gibson }\end{array}$ & Adavi patti & 489 & $\mathrm{H}$ & $R$ & Fever, diuretic \\
\hline Triumfetta rhomboidea Jacq. & Marla benda & 36 & $\mathrm{H}$ & $\mathrm{R}$ & Galactogogue \\
\hline Waltheria indica $\mathrm{L}$. & Nalla benda & 35 & $\mathrm{H}$ & $\mathrm{L}$ & Insect bite \\
\hline Urena lobata L. & Pedda benda & 508 & $\mathrm{H}$ & $R$ & Skin disease \\
\hline \multicolumn{6}{|l|}{ MELASTOMATACEAE } \\
\hline Memecylon umbellatum Burm.f. & Alli & 1034 & $\mathrm{~T}$ & $R$ & Diuretic \\
\hline
\end{tabular}




\section{Suthari et al. - Intracultural Cognizance of Medicinal Plants of Warangal North Forest Division, Northern Telangana, India}

\begin{tabular}{|c|c|c|c|c|c|}
\hline \multicolumn{2}{|l|}{ Botanical Names } & \multirow{2}{*}{$\begin{array}{l}\text { Voucher } \\
\text { Number }\end{array}$} & \multirow[t]{2}{*}{ Habit } & \multirow{2}{*}{$\begin{array}{l}\text { Part(s) } \\
\text { used }\end{array}$} & \multirow[t]{2}{*}{ Ethnic use } \\
\hline Scientific & Vernacular & & & & \\
\hline $\begin{array}{l}\text { Osbeckia stellata Buch.-Ham. } \\
\text { ex Ker Gawl. }\end{array}$ & Adavi gulabi & 1125 & $s$ & $R$ & Abortifacient \\
\hline \multicolumn{6}{|l|}{ MELIACEAE } \\
\hline Azadirachta indica A.Juss. & Vepa & 484 & $\mathrm{~T}$ & $B$ & Stomach-ache \\
\hline Soymida febrifuga (Roxb.) A.Juss. & Somi & 859 & $\mathrm{~T}$ & $\mathrm{~B}$ & Tonic, diuretic \\
\hline \multicolumn{6}{|l|}{ MENISPERMACEAE } \\
\hline Cissampelos pareira L. & Bodhi & 322 & C & $\mathrm{R}$ & Cardiac stimulant \\
\hline Cocculus hirsutus (L.) W.Theob. & Shibbi teega & 1 & $\mathrm{C}$ & $\mathrm{R}$ & Veterinary \\
\hline \multicolumn{6}{|l|}{ MORACEAE } \\
\hline Ficus benghalensis L. & Marri & 309 & $\mathrm{~T}$ & $\mathrm{La}$ & Wounds \\
\hline Ficus hispida L.f. & Bomma medi & 114 & $\mathrm{~T}$ & $\mathrm{~L}$ & Bone fracture \\
\hline Ficus microcarpa L.f. & Juvvi & 974 & $\mathrm{~T}$ & $\mathrm{~B}$ & Leucoderma \\
\hline Ficus racemosa L. & Medi & 565 & $\mathrm{~T}$ & $\mathrm{~L}$ & Bone fracture \\
\hline $\begin{array}{l}\text { Ficus tinctoria subsp. gibbosa (Blume) } \\
\text { Corner }\end{array}$ & \begin{tabular}{|l|} 
Adavi \\
barrenka
\end{tabular} & 936 & $T$ & $B$ & Veterinary \\
\hline Ficus virens Aiton & Banda juvvi & 955 & $\mathrm{~T}$ & $\mathrm{~B}$ & Rheumatism \\
\hline Streblus asper Lour. & Barrenka & 171 & $\mathrm{~T}$ & TS & Tooth brush \\
\hline \multicolumn{6}{|l|}{\begin{tabular}{|l|} 
MORINGACEAE \\
\end{tabular}} \\
\hline Moringa pterygosperma Gaertn. & Munaga & 92 & $\mathrm{~T}$ & $B$ & Aphrodisiac \\
\hline \multicolumn{6}{|l|}{ MYRTACEAE } \\
\hline Syzygium cumini (L.) Skeels & Neredu & 580 & $\mathrm{~T}$ & $\mathrm{~B} / \mathrm{Fr}$ & Tonic, diabetes \\
\hline \multicolumn{6}{|l|}{ NYCTAGINACEAE } \\
\hline Boerhavia diffusa L. & Atika mamidi & 18 & $\mathrm{H}$ & W/R & Fever, tonic \\
\hline \multicolumn{6}{|l|}{ OCHNACEAE } \\
\hline Ochna obtusata DC. & Sonnari & 781 & $\mathrm{~T}$ & $B$ & Veterinary \\
\hline \multicolumn{6}{|l|}{\begin{tabular}{|l|} 
OLEACEAE \\
\end{tabular}} \\
\hline Nyctanthes arbor-tristis L. & Karise & 227 & $\mathrm{~T}$ & $\mathrm{~B}$ & Bone fracture \\
\hline Schrebera swietenioides Roxb. & Mokkam & 1405 & $T$ & $B$ & Veterinary \\
\hline \multicolumn{6}{|l|}{ ORCHIDACEAE } \\
\hline $\begin{array}{l}\text { Vanda tessellata (Roxb.) Hook. } \\
\text { ex G.Don }\end{array}$ & $\begin{array}{l}\text { Kodikalla } \\
\text { chettu }\end{array}$ & 1161 & $E$ & $R$ & Fever \\
\hline \multicolumn{6}{|l|}{ PAPAVARACEAE } \\
\hline Argemone mexicana L. & Mulu puccha & 140 & $\mathrm{H}$ & W & Skin disease \\
\hline \multicolumn{6}{|l|}{ PHYLLANTHACEAE } \\
\hline Breynia retusa (Dennst.) Alston & Purugudu & 1214 & $S$ & $\mathrm{R}$ & Aphrodisiac \\
\hline Bridelia montana (Roxb.) Willd. & Panchotkam & 310 & $\mathrm{~T}$ & $\mathrm{~B}$ & Veterinary \\
\hline Bridelia retusa (L.) A.Juss. & Korra maddi & 772 & $\mathrm{~T}$ & B & Veterinary \\
\hline $\begin{array}{l}\text { Cleistanthus collinus (Roxb.) Benth. } \\
\text { ex Hook.f. }\end{array}$ & Nalla kodisa & 601 & $T$ & \begin{tabular}{|l} 
All \\
parts
\end{tabular} & Homicide, fish stupifying \\
\hline $\begin{array}{l}\text { Glochidion zeylanicum (Gaertn.) } \\
\text { A.Juss. }\end{array}$ & Neeralli & 1047 & $T$ & $B$ & Veterinary \\
\hline $\begin{array}{l}\text { Phyllanthus amarus Schumach. } \\
\text { \& Thonn. }\end{array}$ & Nela usiri & 24 & $\mathrm{H}$ & $W$ & Jaundice \\
\hline
\end{tabular}




\begin{tabular}{|c|c|c|c|c|c|}
\hline \multicolumn{2}{|l|}{ Botanical Names } & \multirow{2}{*}{\begin{tabular}{|l} 
Voucher \\
Number
\end{tabular}} & \multirow[t]{2}{*}{ Habit } & \multirow{2}{*}{$\begin{array}{l}\text { Part(s) } \\
\text { used }\end{array}$} & \multirow[t]{2}{*}{ Ethnic use } \\
\hline Scientific & Vernacular & & & & \\
\hline Phyllanthus emblica L. & Usiri & 602 & $\mathrm{~T}$ & W & Tonic \\
\hline Phyllanthus urinaria L. & Yerra usiri & 1852 & $\mathrm{H}$ & W & Jaundice \\
\hline \multicolumn{6}{|l|}{ PLANTAGINACEAE } \\
\hline Scoparia dulcis L. & Oosari & 519 & $\mathrm{H}$ & W & Menstrual pains \\
\hline \multicolumn{6}{|l|}{ PLUMBAGINACEAE } \\
\hline Plumbago zeylanica L. & Chitramulam & 682 & $\mathrm{H}$ & $R$ & Boils \\
\hline \multicolumn{6}{|l|}{ POACEAE } \\
\hline Bambusa bambos (L.) Voss & Kanka bongu & 521 & $\mathrm{~T}$ & $\mathrm{Se}$ & Food \\
\hline $\begin{array}{l}\text { Cymbopogon flexuosus (Nees } \\
\text { ex Steud.) W.Watson }\end{array}$ & $\begin{array}{l}\text { Adavi nimma } \\
\text { gaddi }\end{array}$ & 425 & $\mathrm{H}$ & $R$ & Tonic \\
\hline \multicolumn{6}{|l|}{ PUTRANJIVACEAE } \\
\hline Putranjiva roxburghii Wall. & Putranjiva & 1048 & $\mathrm{~T}$ & $\mathrm{~B}$ & Bronchitis \\
\hline \multicolumn{6}{|l|}{ RHAMNACEAE } \\
\hline Ventilago maderaspatana Gaertn. & Galivana teega & 849 & C & $\mathrm{B}$ & Anthrax \\
\hline Ziziphus oenopolia (L.) Mill. & Pariki & 256 & $\mathrm{~s}$ & $\mathrm{Fr}$ & Diuretic \\
\hline Ziziphus rugosa Lam. & Enugu pariki & 1643 & $\mathrm{C}$ & $\mathrm{L}$ & Bone fracture \\
\hline Ziziphus xylopyrus (Retz.) Willd. & Gotte & 347 & $\mathrm{~T}$ & $\mathrm{~L}$ & Skin disease \\
\hline \multicolumn{6}{|l|}{ RUBIACEAE } \\
\hline $\begin{array}{l}\text { Catunaregam spinosa (Thunb.) } \\
\text { Tirveng. }\end{array}$ & Manga & 303 & $\mathrm{~T}$ & $\mathrm{Fr}$ & Laxative \\
\hline Ceriscoides turgida (Roxb.) Tirveng. & Kukka elka & 358 & $\mathrm{~T}$ & $\mathrm{~B}$ & Cough \\
\hline Gardenia latifolia Aiton & Pedda karinga & 1224 & $\mathrm{~T}$ & $\mathrm{~B}$ & Rheumatism, veterinary \\
\hline Gardenia resinifera Roth & Karinga & 764 & $\mathrm{~s}$ & $\mathrm{~B}$ & Neck pains \\
\hline Haldina cordifolia (Roxb.) Ridsdale & Bandaru & 422 & $\mathrm{~T}$ & B & Veterinary \\
\hline Ixora arborea Roxb. ex Sm. & Korivi & 1034 & $\mathrm{~T}$ & $\mathrm{~B}$ & Appetite stimulant \\
\hline Mitragyna parvifolia (Roxb.) Korth. & Batta ganapa & 852 & $\mathrm{~T}$ & $\mathrm{~B}$ & Veterinary \\
\hline Morinda pubescens Sm. & Togara mogili & 267 & $\mathrm{~T}$ & $\mathrm{~B}$ & Appetite stimulant, anthrax \\
\hline Paederia foetida L. & Savirela & 1634 & C & $\mathrm{L}$ & Skin disease \\
\hline $\begin{array}{l}\text { Tamilnadia uliginosa (Retz.) Tirveng. } \\
\text { \& Sastre }\end{array}$ & Guvvenka & 450 & $\mathrm{~T}$ & $B$ & Veterinary \\
\hline \multicolumn{6}{|l|}{ RUTACEAE } \\
\hline Aegle marmelos (L.) Corrêa & Maredu & 370 & $\mathrm{~T}$ & $\mathrm{~L} / \mathrm{Fr}$ & Skin disease, digestive \\
\hline Atalantia monophylla DC. & Adavi nimma & 858 & $\mathrm{~T}$ & $\mathrm{R}$ & Paralysis \\
\hline Chloroxylon swietenia DC. & Bilugu & 1046 & $\mathrm{~T}$ & $\mathrm{~B}$ & Skin disease \\
\hline Limonia acidissima L. & Velaga & 741 & $\mathrm{~T}$ & $\mathrm{Fr}$ & Tonic \\
\hline \multicolumn{6}{|l|}{ SALICACEAE } \\
\hline Casearia nigrescens Tue. & Kanmeswaram & 534 & $\mathrm{~T}$ & $B$ & Fish poison \\
\hline Flacourtia indica (Burm.f.) Merr. & Kanregu & 854 & $\mathrm{~T}$ & $\mathrm{~L}$ & Scorpion bite \\
\hline \multicolumn{6}{|l|}{ SAPINDACEAE } \\
\hline Sapindus emarginatus Vahl & Kunkudu & 39 & $\mathrm{~T}$ & $\mathrm{Fr}$ & Hair wash \\
\hline Schleichera oleosa (Lour.) Merr. & Pusuku & 1367 & $\mathrm{~T}$ & $\mathrm{~B}$ & Dyspepsia \\
\hline
\end{tabular}




\section{Suthari et al. - Intracultural Cognizance of Medicinal Plants of Warangal North Forest Division, Northern Telangana, India}

\begin{tabular}{|c|c|c|c|c|c|}
\hline \multicolumn{2}{|l|}{ Botanical Names } & \multirow{2}{*}{$\begin{array}{l}\text { Voucher } \\
\text { Number }\end{array}$} & \multirow[t]{2}{*}{ Habit } & \multirow{2}{*}{$\begin{array}{l}\text { Part(s) } \\
\text { used }\end{array}$} & \multirow[t]{2}{*}{ Ethnic use } \\
\hline Scientific & Vernacular & & & & \\
\hline \multicolumn{6}{|l|}{ SAPOTACEAE } \\
\hline $\begin{array}{l}\text { Madhuca longifolia var. latifolia } \\
\text { (Roxb.) A.Chev. }\end{array}$ & Ippa & 268 & $\mathrm{~T}$ & $\mathrm{Fl} / \mathrm{Se}$ & Dyspepsia, hair oil \\
\hline Manilkara hexandra (Roxb.) Dubard & Pala & 576 & $\mathrm{~T}$ & $\mathrm{Fr}$ & Laxative \\
\hline \multicolumn{6}{|l|}{ SIMAROUBACEAE } \\
\hline Ailanthus excelsa Roxb. & Peda manu & 519 & $\mathrm{~T}$ & $\mathrm{~B}$ & Piles \\
\hline \multicolumn{6}{|l|}{ SMILACACEAE } \\
\hline Smilax perfoliata Lour. & Nageti dumpa & 1601 & $\mathrm{C}$ & $\mathrm{T}$ & Abortifacient \\
\hline \multicolumn{6}{|l|}{ SOLANACEAE } \\
\hline Solanum virginianum $\mathrm{L}$. & Vakudu & 124 & $\mathrm{C}$ & $\mathrm{Fr}$ & Laxative \\
\hline \multicolumn{6}{|l|}{ ULMACEAE } \\
\hline Holoptelea integrifolia Planch. & Nemali nara & 340 & $\mathrm{~T}$ & $\mathrm{~L}$ & Skin disease \\
\hline \multicolumn{6}{|l|}{ VIOLACEAE } \\
\hline $\begin{array}{l}\text { Hybanthus enneaspermus (L.) } \\
\text { F.Muell. }\end{array}$ & Nela kobbari & 97 & $\mathrm{H}$ & $W$ & Aphrodisiac \\
\hline \multicolumn{6}{|l|}{ VITACEAE } \\
\hline Cissus vitiginea $\mathrm{L}$. & Adavi draksha & 734 & $S$ & $\mathrm{Fr}$ & Healing power \\
\hline Leea asiatica (L.) Ridsdale & Velama sandi & 1094 & $\mathrm{~s}$ & $\mathrm{R}$ & Skin disease \\
\hline \multicolumn{6}{|l|}{ ZINGIBERACEAE } \\
\hline Curcuma pseudomontana J.Graham & Adavi pasupu & 956 & $\mathrm{H}$ & $\mathrm{T}$ & Antiseptic \\
\hline
\end{tabular}

ily use "apparent" species (trees and their climbers) which are wild and abundant in the adjoining forests, and (ii) the relatively infrequent use of herbs or "non-apparent" species is reflective of their limited distribution to open areas or secondary forest patches tending towards therophytic phytoclimate.

The number of medicinal plant species reported per village (Table 4) ranged from 15 (Oorattum) to 167 (Mallur). Eighty-two plant species were reported only in one village while 30 species were reported in 9 or more villages (i.e., $\geq 50 \%)$. Each of the 237 plants was scored for shared use (common citation) among the 18 villages. Plants were grouped into shared-use batches that reflected the extent of common plant knowledge (1-3 villages, 4-6 villages, ...16-18 villages) (Figure 2). Results from this analysis show that (i) plant knowledge among the different villages is not uniform but is rather shared by an average of 1 to 3 villages, and (ii) shared knowledge declines over an increasing number of villages, with no single plant species being shared by 16 or more of the 18 villages.

\section{Floristic cognizance}

A total of 237 different plant species were listed as phytomedicines for Koyas across all 18 villages. These were predominantly angiosperms $(233 ; 98.3 \%)$ and a few ferns
(4; $1.7 \%)$, the latter of which were used only in one village (Mallur). Amongst the angiosperms, far more Dicotyledonae species were mentioned (220 species; $94.4 \%$ ). The species used are almost all indigenous (219; 92.4\%) with the few exotics being either planted or naturalized.

The present study found the local people using both indigenous and exotic species, the latter of which are cultivated or naturalized for economic purposes. Some exotic weeds, however, appear inadvertently as escapees in natural forests in areas of biotic disturbance and land-use change (podu [shifting] cultivation). The exotics are normally light-demanding and unacceptable as graze or forage as they are expected to bear more diverse secondary metabolites which aid in herbivory defense. In the present survey, the indigenous flora formed the basis $(92.4 \% ; 219$ species) of ethnobotanical practices of Koyas. The ethnomedicine practiced by Koyas in northern Telangana includes phytomedicines applied to both humans and their pets (Table 3 ) with $84 \%$ of plant species used as human medicine, $13 \%$ as veterinary, and just $3 \%$ for both.

\section{Family-use index}

An index was prepared to investigate the top five plant families which offer the most to the medicinal plants cognitive domain of the ethnic inhabitants of the 18 villages 
Table 3. Breakdown of plant taxonomy, habit, ethnomedicinal use, and nativity by village. All villages are in Warangal North Forest Division of Northern Telangana, India. Villages denoted with an asterisk $\left(^{*}\right)$ are outside the Eturnagaram Wildlife Sanctuary while all others are located within it. Taxonomic groupings: ferns (F), dicots (D), monocots (M), genera $(G)$, species $(S)$. Growth forms: trees $(T)$, shrubs $(S)$, herbs $(H)$, climbers/lianas/epiphytes/stragglers $(C)$. Ethnomedicine: human $(\mathrm{H})$, veterinary $(\mathrm{V})$, both human and veterinary $(\mathrm{H} / \mathrm{V})$. Nativity: indigenous $(\mathrm{I})$, exotic $(E)$.

\begin{tabular}{|l|c|c|c|c|c|c|c|c|c|c|c|c|c|c|}
\hline \multirow{2}{*}{ Villages } & \multicolumn{4}{|c|}{ Taxonomic groupings } & \multicolumn{4}{|c|}{ Growth forms } & \multicolumn{3}{|c|}{ Ethnomedicines } & \multicolumn{2}{c|}{ Nativity } \\
\cline { 2 - 7 } & F & D & M & G & S & T & S & H & C & H & V & H/V & I & E \\
\hline Mallur* & 4 & 153 & 10 & 133 & 167 & 72 & 12 & 51 & 32 & 144 & 19 & 4 & 155 & 12 \\
\hline Kamaram & & 19 & & 18 & 19 & 15 & 1 & 2 & 1 & 16 & 1 & 2 & 17 & 2 \\
\hline Venkatapuram & & 28 & & 26 & 28 & 18 & 1 & 5 & 4 & 22 & 5 & 1 & 26 & 2 \\
\hline Chinnaboinapally & & 41 & 1 & 39 & 42 & 11 & 1 & 22 & 8 & 37 & 4 & 1 & 36 & 6 \\
\hline Shapally & & 102 & 5 & 92 & 107 & 50 & 8 & 34 & 15 & 97 & 8 & 2 & 97 & 10 \\
\hline Dodla & & 101 & 4 & 94 & 105 & 45 & 6 & 38 & 16 & 93 & 11 & 1 & 93 & 12 \\
\hline Malyala & & 33 & 1 & 33 & 34 & 19 & & 8 & 7 & 31 & 3 & & 31 & 3 \\
\hline Kondai & & 65 & 3 & 60 & 68 & 34 & 3 & 22 & 9 & 59 & 8 & 1 & 63 & 5 \\
\hline Ailapur & & 57 & 2 & 56 & 59 & 23 & 2 & 22 & 12 & 51 & 8 & & 53 & 6 \\
\hline Yelishettipally & & 19 & 2 & 21 & 21 & 12 & 2 & 6 & 1 & 18 & 2 & 1 & 17 & 4 \\
\hline Banojibandam & & 21 & & 21 & 21 & 8 & 4 & 5 & 4 & 19 & 1 & 1 & 20 & 1 \\
\hline Chalpaka & & 24 & 1 & 25 & 25 & 14 & & 6 & 5 & 23 & 2 & & 24 & 1 \\
\hline Allamvari Ghanpur & & 36 & & 34 & 36 & 11 & & 19 & 6 & 32 & 3 & 1 & 30 & 6 \\
\hline Narlapur & & 100 & 5 & 94 & 105 & 49 & 7 & 35 & 14 & 91 & 11 & 3 & 95 & 10 \\
\hline Medaram & & 40 & & 37 & 40 & 27 & 2 & 8 & 3 & 30 & 7 & 3 & 37 & 3 \\
\hline Oorattum & & 15 & & 15 & 15 & 10 & 1 & & 3 & 14 & 1 & & 14 & 1 \\
\hline Gonepally & & 81 & 5 & 80 & 86 & 36 & 5 & 28 & 18 & 78 & 7 & 1 & 80 & 6 \\
\hline Gandhinagar* & & 21 & & 19 & 21 & 11 & 1 & 6 & 3 & 17 & 3 & 1 & 18 & 3 \\
\hline
\end{tabular}

Table 4. Reports of plant species usage as Koya ethnomedicine for each of 18 villages within or near Eturnagaram Wildlife Sanctuary in Warangal North Forest Division of Northern Telangana, India. Villages: (1) Mallur; (2) Kamaram; (3) Venkatapuram; (4) Chinnaboinapally; (5) Shapally; (6) Dodla; (7) Malyala; (8) Kondai; (9) Ailapur; (10) Yelishettipally; (11) Banojibandam; (12) Chalpaka; (13) Allamvari Ghanpur; (14) Narlapur; (15) Medaram; (16) Oorattum; (17) Gonepally; (18) Gandhinagar. Villages 1 and 18 are outside the Eturnagaram Wildlife Sanctuary.

\begin{tabular}{|c|c|c|c|c|c|c|c|c|c|c|c|c|c|c|c|c|c|c|}
\hline \multirow[t]{2}{*}{ Scientific name } & \multicolumn{18}{|c|}{ Villages } \\
\hline & 1 & 2 & 3 & 4 & 5 & 6 & 7 & 8 & 9 & 10 & 11 & 12 & 13 & 14 & 15 & 16 & 17 & 18 \\
\hline \multicolumn{19}{|l|}{ ACANTHACEAE } \\
\hline $\begin{array}{l}\text { Andrographis paniculata (Burm.f.) Wall. } \\
\text { ex Nees }\end{array}$ & $X$ & & & $\mathrm{X}$ & $\mathrm{X}$ & $\mathrm{X}$ & $\mathrm{X}$ & $\mathrm{X}$ & $\mathrm{X}$ & & & $\mathrm{X}$ & $\mathrm{X}$ & $\mathrm{X}$ & & & $\mathrm{X}$ & \\
\hline Barleria cristata L. & $\mathrm{X}$ & & & & $\mathrm{X}$ & & & & & & & & & $X$ & & & $\mathrm{X}$ & \\
\hline Barleria montana Nees & $\mathrm{X}$ & & & & & & & & & & & & & & & & & \\
\hline Barleria prionitis L. & $\mathrm{X}$ & & & & & & & & & & & & & & & & & \\
\hline Hemigraphis latebrosa (Roth) Nees & $\mathrm{X}$ & & & & & & & & & & & & & & & & & \\
\hline Hygrophila auriculata (Schumach.) Heine & & & & $\mathrm{X}$ & & $\mathrm{X}$ & & $\mathrm{X}$ & & & & & $\mathrm{X}$ & & & & & \\
\hline Justicia betonica L. & $\mathrm{X}$ & & & & $X$ & $\mathrm{X}$ & & $\mathrm{X}$ & & & & & & $x$ & & & $x$ & \\
\hline Lepidagathis cristata Willd. & $\mathrm{X}$ & & & & $\mathrm{X}$ & $\mathrm{x}$ & & & $\mathrm{X}$ & & & & & $\mathrm{x}$ & & & $\mathrm{x}$ & \\
\hline Peristrophe paniculata (Forssk.) Brummitt & $\mathrm{X}$ & & & & $\mathrm{X}$ & $\mathrm{X}$ & & & & & & & & & & & & \\
\hline \multicolumn{19}{|l|}{ AMARANTHACEAE } \\
\hline Achyranthes aspera L. & $\mathrm{X}$ & & & $X$ & $\mathrm{X}$ & $\mathrm{X}$ & & $\mathrm{X}$ & $\mathrm{X}$ & & & & $X$ & $\mathrm{x}$ & & & $x$ & \\
\hline
\end{tabular}




\section{Suthari et al. - Intracultural Cognizance of Medicinal Plants of Warangal North Forest Division, Northern Telangana, India}

\begin{tabular}{|c|c|c|c|c|c|c|c|c|c|c|c|c|c|c|c|c|c|c|}
\hline \multirow[t]{2}{*}{ Scientific name } & \multicolumn{18}{|c|}{ Villages } \\
\hline & 1 & 2 & 3 & 4 & 5 & 6 & 7 & 8 & 9 & 10 & 11 & 12 & 13 & 14 & 15 & 16 & 17 & 18 \\
\hline Aerva lanata (L.) Juss. & $x$ & & & $\mathrm{X}$ & $\mathrm{X}$ & $\mathrm{X}$ & & $\mathrm{X}$ & $\mathrm{X}$ & $\mathrm{X}$ & & & $x$ & $x$ & & & $\mathrm{x}$ & \\
\hline Aerva sanguinolenta (L.) Blume & $\mathrm{X}$ & & & $\mathrm{X}$ & $\mathrm{X}$ & $\mathrm{X}$ & & $\mathrm{X}$ & $\mathrm{X}$ & & & & & & & & & \\
\hline Amaranthus spinosus L. & $\mathrm{X}$ & & & & $\mathrm{X}$ & $X$ & & & & $\mathrm{X}$ & & & & $x$ & & & & \\
\hline Celosia argentea L. & & & & & & $x$ & & & $x$ & & & & & & & & & \\
\hline Pupalia lappacea (L.) Juss. & $\mathrm{X}$ & & & & & & & & & & & & & & & & & \\
\hline \multicolumn{19}{|l|}{ ANACARDIACEAE } \\
\hline Buchanania axillaris (Desr.) Ramamoorthy & & & $\mathrm{X}$ & & & & & & & & & & & $x$ & $\mathrm{X}$ & & $\mathrm{X}$ & \\
\hline $\begin{array}{l}\text { Buchanania cochinchinensis (Lour.) } \\
\text { M.R.Almeida }\end{array}$ & $\mathrm{X}$ & $X$ & & & $X$ & $\mathrm{X}$ & & $\mathrm{X}$ & & & & & & $x$ & & & $\mathrm{X}$ & \\
\hline Lannea coromandelica (Houtt.) Merr. & $\mathrm{X}$ & $\mathrm{X}$ & & $\mathrm{X}$ & $\mathrm{X}$ & $\mathrm{X}$ & & $\mathrm{X}$ & $\mathrm{X}$ & & & & $x$ & $\mathrm{x}$ & $\mathrm{X}$ & & & \\
\hline Mangifera indica L. & $\mathrm{X}$ & $\mathrm{X}$ & & & $\mathrm{X}$ & $\mathrm{X}$ & & & $\mathrm{X}$ & & & & & $\mathrm{X}$ & & & $\mathrm{x}$ & $X$ \\
\hline Semecarpus anacardium L.f. & $\mathrm{X}$ & & $\mathrm{X}$ & & $\mathrm{X}$ & $\mathrm{X}$ & & $\mathrm{X}$ & & & & & & & $\mathrm{X}$ & & & \\
\hline \multicolumn{19}{|l|}{ ANNONACEAE } \\
\hline Miliusa tomentosa (Roxb.) J.Sinclair & & & $\mathrm{X}$ & & & & & & & & & & & & $\mathrm{X}$ & & & \\
\hline Polyalthia cerasoides (Roxb.) Bedd. & $\mathrm{X}$ & & & & $\mathrm{X}$ & $\mathrm{X}$ & & & $\mathrm{X}$ & & & & & $x$ & & & $\mathrm{x}$ & \\
\hline \multicolumn{19}{|l|}{ APOCYNACEAE } \\
\hline Anodendron paniculatum A.DC. & $\mathrm{X}$ & & & & & & & & & & & & & & & & & \\
\hline Calotropis gigantea (L.) Dryand. & & & & & & $\mathrm{X}$ & & & & $\mathrm{X}$ & & & & & & & & \\
\hline Cyptolepis dubia (Burm.f.) M.R.Almeida & & & $\mathrm{X}$ & & & & & & & & & & & & $\mathrm{X}$ & & & \\
\hline Hemidesmus indicus (L.) R.Br. ex Schult. & $\mathrm{X}$ & $\mathrm{X}$ & $\mathrm{X}$ & $\mathrm{X}$ & $\mathrm{x}$ & $\mathrm{x}$ & $\mathrm{X}$ & $\mathrm{X}$ & $\mathrm{X}$ & & & $\mathrm{X}$ & $x$ & & $\mathrm{X}$ & & & $\mathrm{X}$ \\
\hline $\begin{array}{l}\text { Hemidesmus indicus var. pubescens } \\
\text { (Wight \& Arn.) Hook.f. }\end{array}$ & & & & & & & & & & & & & & & & & $\mathrm{X}$ & \\
\hline Holarrhena pubescens Wall ex G.Don & $\mathrm{X}$ & $X$ & $X$ & & $\mathrm{X}$ & $X$ & $\mathrm{X}$ & $\mathrm{X}$ & $X$ & $X$ & $\mathrm{X}$ & $\mathrm{X}$ & & $X$ & $\mathrm{X}$ & & & \\
\hline Pergularia daemia (Forssk.) Chiov. & & & $\mathrm{X}$ & & & & & & & & & & & $x$ & $\mathrm{X}$ & & $\mathrm{X}$ & \\
\hline \multicolumn{19}{|l|}{ ARACEAE } \\
\hline Pistia stratiotes L. & & & & & & & & & & & & & & & & & & $\mathrm{X}$ \\
\hline \multicolumn{19}{|l|}{ ARECACEAE } \\
\hline Phoenix loureiroi Kunth & $\mathrm{X}$ & & & & $\mathrm{X}$ & $\mathrm{x}$ & & & & & & & & $x$ & & & $\mathrm{X}$ & \\
\hline \multicolumn{19}{|l|}{ ARISTOLOCHIACEAE } \\
\hline Aristolochia indica L. & $\mathrm{X}$ & & & & $\mathrm{X}$ & $\mathrm{X}$ & & & & & $\mathrm{X}$ & & & $x$ & & & $\mathrm{X}$ & \\
\hline \multicolumn{19}{|l|}{ ASPARAGACEAE } \\
\hline Asparagus gonoclados Baker & & & & & $\mathrm{X}$ & & & & & & & & & & & & & \\
\hline Asparagus racemosus Willd. & $\mathrm{X}$ & & & & & & & & & & & & & & & & & \\
\hline \multicolumn{19}{|l|}{ ASTERACEAE } \\
\hline Blumea bifoliata (L.) DC. & $\mathrm{X}$ & & & & $X$ & & & & & & & & & $x$ & & & $\mathrm{X}$ & \\
\hline Blumea virens DC. & $\mathrm{X}$ & & & & $\mathrm{X}$ & & & & & & & & & & & & & \\
\hline Pentanema indicum (L.) Ling & $\mathrm{X}$ & & & & & & & & & & & & & & & & & \\
\hline Sphaeranthus indicus L. & $\mathrm{X}$ & & & $\mathrm{X}$ & $X$ & $x$ & $\mathrm{X}$ & $\mathrm{X}$ & $\mathrm{X}$ & & & $\mathrm{X}$ & $x$ & $x$ & & & $\mathrm{X}$ & \\
\hline Tridax procumbens (L.) L. & $\mathrm{X}$ & & & & & & & & $\mathrm{X}$ & & & & & & & & & \\
\hline Vernonia cinerea (L.) Less. & $\mathrm{X}$ & & & $\mathrm{X}$ & $X$ & $x$ & & $\mathrm{X}$ & $x$ & & & & $x$ & $x$ & & & $\mathrm{X}$ & \\
\hline $\begin{array}{l}\text { Vernonia cinerea var. parviflora (Reinw. } \\
\text { ex Blume) DC. }\end{array}$ & $\mathrm{X}$ & & & & & $x$ & & & & & & & & & & & & \\
\hline
\end{tabular}




\begin{tabular}{|c|c|c|c|c|c|c|c|c|c|c|c|c|c|c|c|c|c|c|c|}
\hline \multirow[t]{2}{*}{ Scientific name } & \multicolumn{19}{|c|}{ Villages } \\
\hline & 1 & 2 & 3 & 4 & 5 & 6 & 7 & 8 & 9 & 1 & & 11 & 12 & 13 & 14 & 15 & 16 & 17 & 18 \\
\hline \multicolumn{20}{|l|}{ BIGNONIACEAE } \\
\hline Millingtonia hortensis L.f. & $x$ & & & & & & & & & & & & & & & & & & \\
\hline Oroxylum indicum (L.) Kurz & $x$ & & & & & & & & & & & & & & & & & & \\
\hline Stereospermum chelonoides (L.f.) DC. & $x$ & & & & & & & & & & & & & & & $x$ & & & \\
\hline \multicolumn{20}{|l|}{ BOMBACACEAE } \\
\hline Bombax ceiba L. & & $x$ & & & & & & & & & & & & & & & & & \\
\hline \multicolumn{20}{|l|}{ BORAGINACEAE } \\
\hline Coldenia procumbens L. & & & & & & & & & & & & & & & $x$ & & & $x$ & \\
\hline Cordia dichotoma G.Forst. & $\mathrm{X}$ & & & & & & & & & & & & & & & & & $x$ & \\
\hline Cordia macleodii Hook.f. \& Thomson & $\mathrm{X}$ & & & & & & & & & & & & & & & & & & \\
\hline Ehretia laevis Roxb. & $x$ & & & & & & & & & & & & & & & & & & \\
\hline Trichodesma indicum (L.) Lehm. & & & & $x$ & & $x$ & & $x$ & $x$ & & & & & $x$ & & & & & \\
\hline \multicolumn{20}{|l|}{ BURSERACEAE } \\
\hline Boswellia serrata Roxb. ex Colebr. & $x$ & & & & $x$ & $x$ & $\mathrm{x}$ & $\mathrm{x}$ & & 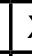 & & $\underline{x}$ & & & $\mathrm{X}$ & & $x$ & & \\
\hline Garuga pinnata Roxb. & & & & & & & & & & & & & & & $x$ & & & & \\
\hline \multicolumn{20}{|l|}{ CAPPARACEAE } \\
\hline Capparis sepiaria L. & & & & & & & & & & & & & & & & & & $\mathrm{x}$ & \\
\hline Capparis zeylanica L. & $\mathrm{X}$ & & & & $\mathrm{X}$ & $x$ & & & & & & $x$ & & & $x$ & & $x$ & $x$ & \\
\hline \multicolumn{20}{|l|}{ CARYOPHYLLACEAE } \\
\hline Polycarpaea corymbosa (L.) Lam. & $\mathrm{X}$ & & & & & $\mathrm{x}$ & & & & & & & & & $x$ & & & $\mathrm{X}$ & \\
\hline \multicolumn{20}{|l|}{ CELASTRACEAE } \\
\hline Cassine glauca (Rottb.) Kuntze & $\mathrm{X}$ & & & & $\mathrm{X}$ & & & & & & & & & & $x$ & & & & \\
\hline $\begin{array}{l}\text { Gymnosporia emarginata (Willd.) } \\
\text { Thwaites }\end{array}$ & $x$ & & & & $x$ & $x$ & & & $x$ & $>$ & & & & & $x$ & & & & \\
\hline \multicolumn{20}{|l|}{ CLEOMACEAE } \\
\hline Cleome viscosa $\mathrm{L}$. & $x$ & & & & $x$ & $x$ & & & $x$ & & & & & & $x$ & & & $x$ & \\
\hline \multicolumn{20}{|l|}{ COMBRETACEAE } \\
\hline $\begin{array}{l}\text { Anogeissus acuminata (Roxb. ex DC.) } \\
\text { Guill., Perr. \& A.Rich. }\end{array}$ & & & & & & $\mathrm{x}$ & & & & & & & & & & & & & \\
\hline Combretum albidum G.Don & $\mathrm{x}$ & & & & & & & & & & & & & & & & & & \\
\hline Combretum roxburghii Spreng. & $\mathrm{x}$ & & & $x$ & $x$ & & $x$ & $\mathrm{x}$ & $x$ & & & & & $\mathrm{X}$ & $x$ & & & $\mathrm{X}$ & $x$ \\
\hline Combretum latifolium Blume & $\mathrm{x}$ & & & & & & & & & & & & & & & & & & \\
\hline Getonia floribunda Roxb. & & & & $\mathrm{x}$ & & $x$ & & $x$ & $x$ & & & & & $x$ & & & & & \\
\hline $\begin{array}{l}\text { Terminalia arjuna (Roxb. ex DC.) Wight } \\
\text { \& Arn. }\end{array}$ & $x$ & & & & $x$ & $x$ & & $x$ & & & & & & & $x$ & & & & $x$ \\
\hline Terminalia bellirica (Gaertn.) Roxb. & $x$ & & & & $x$ & $\mathrm{x}$ & $\mathrm{x}$ & $\mathrm{x}$ & $\mathrm{X}$ & & & & $x$ & & & & $x$ & & \\
\hline Terminalia chebula Retz. & & & & & $x$ & & & & & & & & & & & & & & \\
\hline \multicolumn{20}{|l|}{ CONVOLVULACEAE } \\
\hline $\begin{array}{l}\text { Ipomoea coptica (L.) Roth ex Roem. } \\
\text { \& Schult. }\end{array}$ & $x$ & & & & & & & & & & & & & & & & & & \\
\hline Ipomoea eriocarpa R.Br. & $x$ & & & & & & & & & & & & & & & & & & \\
\hline
\end{tabular}




\section{Suthari et al. - Intracultural Cognizance of Medicinal Plants of Warangal North Forest Division, Northern Telangana, India}

\begin{tabular}{|c|c|c|c|c|c|c|c|c|c|c|c|c|c|c|c|c|c|c|}
\hline \multirow[t]{2}{*}{ Scientific name } & \multicolumn{18}{|c|}{ Villages } \\
\hline & 1 & 2 & 3 & 4 & 5 & 6 & 7 & 8 & 9 & 10 & 11 & 12 & 13 & 14 & 15 & 16 & 17 & 18 \\
\hline Ipomoea hederifolia L. & $x$ & & & & & & & & & & & & & & & & & \\
\hline Ipomoea obscura (L.) Ker Gawl. & $\mathrm{x}$ & & & & & & & & & & & & & & & & & \\
\hline Merremia hederacea (Burm.f.) Hallier f. & $\mathrm{x}$ & & & & $\mathrm{x}$ & $\mathrm{x}$ & & & $\mathrm{x}$ & & & & & $\mathrm{x}$ & & & & \\
\hline Rivea hypocrateriformis Choisy & $x$ & & & $\mathrm{x}$ & & & $x$ & & & & & $\mathrm{x}$ & & & & $\mathrm{x}$ & & $x$ \\
\hline \multicolumn{19}{|l|}{ CORNACEAE } \\
\hline Alangium salviifolium (L.f.) Wangerin. & $x$ & & & $\mathrm{x}$ & $\mathrm{x}$ & $\mathrm{X}$ & $\mathrm{x}$ & $\mathrm{X}$ & $x$ & & & $\mathrm{x}$ & $\mathrm{x}$ & $x$ & & $x$ & $\mathrm{X}$ & \\
\hline \multicolumn{19}{|l|}{ CUCURBITACEAE } \\
\hline Diplocyclos palmatus (L.) C.Jeffrey & & & & & & $x$ & & & & & & & & & & & & \\
\hline Momordica charantia L. & & & & & & $x$ & & & & & & & & & & & & \\
\hline Momordica dioica Roxb. ex Willd. & & & & & & & & & & & & & & & & $\mathrm{x}$ & & \\
\hline \multicolumn{19}{|l|}{ CYPERACEAE } \\
\hline Cyperus rotundus $\mathrm{L}$. & $x$ & & & $\mathrm{x}$ & $\mathrm{x}$ & $x$ & & $x$ & $x$ & & & & & $x$ & & & $x$ & \\
\hline Scleria levis Retz. & $x$ & & & & & & & & & & & & & & & & & \\
\hline \multicolumn{19}{|l|}{ DILLENIACEAE } \\
\hline Dillenia pentagyna Roxb. & $x$ & & & & & & & & & & & & & $\mathrm{x}$ & $\mathrm{x}$ & & & \\
\hline \multicolumn{19}{|l|}{ DIOSCOREACEAE } \\
\hline Dioscorea bulbifera L. & $x$ & & & & & & & & & & & & & & & & $\mathrm{X}$ & \\
\hline \multicolumn{19}{|l|}{ EBENACEAE } \\
\hline Diospyros chloroxylon Roxb. & $x$ & & $x$ & & $x$ & $x$ & & $x$ & $x$ & & & & & $x$ & $\mathrm{x}$ & & $\mathrm{x}$ & \\
\hline Diospyros malabarica (Desr.) Kostel. & $x$ & & & & & & & & & & & & & & & & & \\
\hline Diospyros melanoxylon Roxb. & $x$ & & $x$ & & $\mathrm{x}$ & $x$ & $\mathrm{x}$ & $\mathrm{x}$ & $x$ & & & $\mathrm{X}$ & & $\mathrm{x}$ & $x$ & & & $x$ \\
\hline Diospyros montana Roxb. & & $x$ & & & & & & & & & & & & & & & & $\mathrm{x}$ \\
\hline \multicolumn{19}{|l|}{ ERYTHROXYLACEAE } \\
\hline Erythroxylum monogynum Roxb. & & & & & & & & & & & & & & & & & $\mathrm{x}$ & \\
\hline \multicolumn{19}{|l|}{ EUPHORBIACEAE } \\
\hline Acalypha indica L. & & & & & & & & & & $\mathrm{X}$ & $\mathrm{X}$ & & & & $\mathrm{x}$ & & & $\mathrm{x}$ \\
\hline Euphorbia hirta L. & $x$ & & $x$ & $\mathrm{x}$ & $\mathrm{x}$ & $x$ & & $x$ & $x$ & & & & $\mathrm{x}$ & $x$ & $\mathrm{x}$ & & $\mathrm{x}$ & \\
\hline Euphorbia nivulia Buch.-Ham. & & & & & $\mathrm{X}$ & & & & & & & & & & & & & \\
\hline Mallotus philippensis (Lam.) Müll.Arg. & $x$ & & & & $\mathrm{x}$ & $x$ & & & & & & & & & & & & \\
\hline Microstachys chamaelea (L.) Müll.Arg. & $x$ & & & & & & & & & & & & & & & & & \\
\hline Tragia involucrata L. & $x$ & & & & & & & & & & & & & & & & & \\
\hline \multicolumn{19}{|l|}{ FABACEAE } \\
\hline Abrus precatorius $\mathrm{L}$. & $x$ & & & $\mathrm{x}$ & $\mathrm{x}$ & $x$ & & $\mathrm{x}$ & $x$ & $\mathrm{x}$ & $\mathrm{X}$ & & & $\mathrm{x}$ & & & $\mathrm{x}$ & \\
\hline Acacia chundra (Rottler) Willd. & & & & & & & & & & & & & & & & $\mathrm{x}$ & & \\
\hline Acacia nilotica (L.) Willd. ex Del. & & & & & & & & & & & & & & $x$ & & & & \\
\hline Albizia odoratissima (L.f.) Benth. & $x$ & & & & $\mathrm{X}$ & $x$ & & & & & & & & $x$ & & & $\mathrm{x}$ & \\
\hline Bauhinia malabarica Roxb. & $x$ & & & & $x$ & & & & & & & & & $\mathrm{X}$ & & & & \\
\hline Bauhinia racemosa Lam. & $x$ & & & & $x$ & & & & & & & & & $x$ & & & $\mathrm{x}$ & \\
\hline Bauhinia semla Wunderlin & $x$ & & & & & & & & & & & & & & & & & \\
\hline Butea superba Roxb. & $x$ & & & $\mathrm{x}$ & $\mathrm{x}$ & $x$ & $\mathrm{x}$ & $\mathrm{x}$ & & & & $\mathrm{x}$ & $x$ & $\mathrm{x}$ & & & $\mathrm{x}$ & \\
\hline
\end{tabular}




\begin{tabular}{|c|c|c|c|c|c|c|c|c|c|c|c|c|c|c|c|c|c|c|}
\hline \multirow[t]{2}{*}{ Scientific name } & \multicolumn{18}{|c|}{ Villages } \\
\hline & 1 & 2 & 3 & 4 & 5 & 6 & 7 & 8 & 9 & 10 & 11 & 12 & 13 & 14 & 15 & 16 & 17 & 18 \\
\hline Canavalia cathartica Thouars & $x$ & & & & & & & & & & & & & & & & & \\
\hline Cassia fistula L. & $x$ & & & $X$ & $x$ & $x$ & $x$ & $\mathrm{X}$ & $x$ & & & $x$ & $x$ & $x$ & & & $\mathrm{x}$ & $x$ \\
\hline Crotalaria albida B.Heyne ex Roth & $x$ & & & & & & & & & & & & & & & & & \\
\hline Crotalaria ramosissima Roxb. & & & & & & $\mathrm{x}$ & & & & & & & & & & & & \\
\hline Crotalaria verrucosa $\mathrm{L}$. & & & & & & & & & & & & & & & & & $x$ & \\
\hline $\begin{array}{l}\text { Dalbergia lanceolaria subsp. paniculata } \\
\text { (Roxb.) Thoth. }\end{array}$ & $x$ & & $x$ & & $\bar{x}$ & $\bar{x}$ & & $x$ & & $\mathrm{x}$ & & & & $x$ & $x$ & & $x$ & \\
\hline Dalbergia volubilis Roxb. & $x$ & & & & & & & & & & & & & & & & & \\
\hline Derris scandens (Roxb.) Benth. & $x$ & & & & $x$ & $x$ & & & $x$ & & & & & $x$ & & & $x$ & \\
\hline Desmodium gangeticum (L.) DC. & $x$ & & & & $x$ & $\mathrm{x}$ & & & & & & & & & & & & \\
\hline Desmodium triflorum (L.) DC. & $x$ & & & & $x$ & & & & & & & & & $x$ & & & $x$ & \\
\hline Desmodium velutinum (Willd.) DC. & $x$ & & & & & & & & & & & & & & & & & \\
\hline Dichrostachys cinerea (L.) Wight \& Arn. & & & & & $x$ & & & & & & & & & $x$ & & & $x$ & \\
\hline Erythrina suberosa Roxb. & $x$ & $\mathrm{x}$ & & & $x$ & & & & & & $x$ & & & & & & & \\
\hline Flemingia macrophylla (Willd.) Merr. & $x$ & & & & & & & & & & $x$ & & & & & & & \\
\hline Flemingia strobilifera (L.) W.T.Aiton & $x$ & & & & & & & & & & & & & & & & & \\
\hline Hardwickia binata Roxb. & & & & & & & & & & & & & & $x$ & & & & \\
\hline Indigofera cassioides Rottler ex DC. & $x$ & & & & $x$ & $x$ & & & $\mathrm{x}$ & & & & & & & & & \\
\hline Mimosa hamata Willd. & & & & & & & & & & & $x$ & & & & & & $x$ & \\
\hline Mucuna pruriens (L.) DC. & $\mathrm{x}$ & & & $\mathrm{x}$ & $x$ & $\bar{x}$ & & $\mathrm{x}$ & $\mathrm{x}$ & & $x$ & & $\mathrm{x}$ & $\mathrm{x}$ & & $x$ & $x$ & \\
\hline Phyllodium pulchellum (L.) Desv. & $\mathrm{x}$ & & & & $x$ & & & & & & $x$ & & & $\mathrm{x}$ & & & $x$ & \\
\hline Pongamia pinnata (L.) Pierre & $\mathrm{x}$ & & & & & $\mathrm{x}$ & & & & $\mathrm{x}$ & & & & $\mathrm{x}$ & & & $\mathrm{x}$ & \\
\hline Pueraria tuberosa (Willd.) DC. & $x$ & & & & & & & & & & $x$ & & & $x$ & & & & \\
\hline Senna alata (L.) Roxb. & & $x$ & & & & $x$ & & & & $\mathrm{x}$ & $x$ & & & & $\mathrm{x}$ & & & \\
\hline Senna occidentalis (L.) Link & $x$ & & & $\mathrm{x}$ & $x$ & $x$ & $x$ & $x$ & & & & & $\mathrm{x}$ & $\mathrm{x}$ & & & & $x$ \\
\hline Senna tora (L.) Roxb. & $x$ & & & $x$ & $x$ & $\mathrm{x}$ & $x$ & $\mathrm{x}$ & & & & & $\mathrm{x}$ & $x$ & & & & $x$ \\
\hline Sophora velutina Lindl. & $\mathrm{x}$ & & & & & & & & & & & & & & & & & \\
\hline Tamarindus indica L. & $x$ & & & & $x$ & $\mathrm{x}$ & & & & & & & & $x$ & & & $\mathrm{x}$ & \\
\hline Tephrosia purpurea (L.) Pers. & & & $x$ & & & & & & & & & & & & $\mathrm{x}$ & & & $\mathrm{x}$ \\
\hline Xylia xylocarpa (Roxb.) Taub. & $x$ & & $x$ & & $x$ & $x$ & $x$ & $\mathrm{x}$ & $\mathrm{x}$ & & $x$ & & & $x$ & $\mathrm{x}$ & & $x$ & \\
\hline \multicolumn{19}{|l|}{ FERNS AND FERN ALLIES } \\
\hline Ceratopteris thalictroides (L.) Brongn. & $x$ & & & & & & & & & & & & & & & & & \\
\hline Cyclosorus parasiticus (L.) Farw. & $\mathrm{x}$ & & & & & & & & & & & & & & & & & \\
\hline Lygodium flexuosum (L.) Sw. & $x$ & & & & & & & & & & & & & & & & & \\
\hline Selaginella bryopteris (L.) Baker & $\mathrm{x}$ & & & & & & & & & & & & & & & & & \\
\hline \multicolumn{19}{|l|}{ GISEKIACEAE } \\
\hline Gisekia pharnaceoides L. & & & & $\mathrm{x}$ & & $\bar{x}$ & & $\mathrm{x}$ & & & $\mathrm{x}$ & & & & & & & \\
\hline \multicolumn{19}{|l|}{ HYPOXIDACEAE } \\
\hline Curculigo orchioides Gaertn. & $x$ & & & & $x$ & $\mathrm{x}$ & & $\mathrm{x}$ & & $\mathrm{x}$ & & & & $\mathrm{x}$ & & & $\mathrm{x}$ & \\
\hline \multicolumn{19}{|l|}{ LAMIACEAE } \\
\hline Anisomeles malabarica (L.) R.Br. ex Sims & $\mathrm{x}$ & & & & & & & & & & & & & $\mathrm{x}$ & & & & \\
\hline
\end{tabular}




\section{Suthari et al. - Intracultural Cognizance of Medicinal Plants of Warangal North Forest Division, Northern Telangana, India}

\begin{tabular}{|c|c|c|c|c|c|c|c|c|c|c|c|c|c|c|c|c|c|c|}
\hline \multirow[t]{2}{*}{ Scientific name } & \multicolumn{18}{|c|}{ Villages } \\
\hline & 1 & 2 & 3 & 4 & 5 & 6 & 7 & 8 & 9 & 10 & 11 & 12 & 13 & 14 & 15 & 16 & 17 & 18 \\
\hline Gmelina arborea Roxb. & $x$ & & & & & & & & & & & & & $x$ & & & & \\
\hline Leucas aspera (Willd.) Link & & & & & & $\mathrm{x}$ & & & $x$ & & & & & & $\mathrm{x}$ & & & \\
\hline Leucas decemdentata (Willd.) Sm. & $x$ & & & & & & & & & & & & & & & & & \\
\hline Ocimum americanum $\mathrm{L}$. & $x$ & & & $\mathrm{x}$ & $x$ & $\mathrm{X}$ & & & & & & & $\mathrm{x}$ & & & & & \\
\hline Plectranthus mollis (Aiton) Spreng. & $\mathrm{x}$ & & & & $\mathrm{x}$ & $\mathrm{x}$ & & & & & $\mathrm{x}$ & & & $\mathrm{x}$ & & & & \\
\hline Symphorema involucratum Roxb. & & & & & & & & & & & & & & & & & $x$ & \\
\hline Tectona grandis L.f. & & & & & & $x$ & $\mathrm{x}$ & $\mathrm{x}$ & & & & $\mathrm{x}$ & & & & & & \\
\hline \multicolumn{19}{|l|}{ LAURACEAE } \\
\hline Litsea glutinosa (Lour.) C.B. Rob. & $x$ & & & & $\mathrm{X}$ & $x$ & & & & & & & & & & & & \\
\hline \multicolumn{19}{|l|}{ LECYTHIDACEAE } \\
\hline Barringtonia acutangula (L.) Gaertn. & & & & & & $x$ & & & & & $\mathrm{x}$ & & & & & & $x$ & \\
\hline Careya arborea Roxb. & & & $\mathrm{x}$ & & & & & & & & & & & & $x$ & & & $x$ \\
\hline \multicolumn{19}{|l|}{ LINDERNIACEAE } \\
\hline Lindernia ciliata (Colsm.) Pennell & $x$ & & & & & & & & & & & & & & & & & \\
\hline \multicolumn{19}{|l|}{ LOGANIACEAE } \\
\hline Strychnos nux-vomica L. & $x$ & & & $x$ & $x$ & $x$ & $x$ & $x$ & $x$ & & & $\mathrm{x}$ & $x$ & $x$ & & & $x$ & $x$ \\
\hline Strychnos potatorum L.f. & & & $\mathrm{x}$ & & & & & & & & & & & & $x$ & & $x$ & \\
\hline \multicolumn{19}{|l|}{ LORANTHACEAE } \\
\hline Dendrophthoe falcata (L.f.) Ettingsh. & & & & & & & & & & & & & & & & & $x$ & \\
\hline \multicolumn{19}{|l|}{ LYTHRACEAE } \\
\hline Lagerstroemia parviflora Roxb. & $x$ & & $x$ & & $x$ & $x$ & & $\mathrm{x}$ & $\mathrm{x}$ & & & & & $\mathrm{x}$ & $x$ & & & \\
\hline Woodfordia fruticosa (L.) Kurz & $x$ & & & & $\mathrm{X}$ & & & & & & & & & $\mathrm{x}$ & & & $x$ & \\
\hline \multicolumn{19}{|l|}{ MALVACEAE } \\
\hline Abutilon indicum (L.) Sweet & $x$ & & & & $\mathrm{x}$ & $x$ & & & $x$ & & & & & $x$ & & & $x$ & \\
\hline Eriolaena hookeriana Wight \& Arn. & & & & & & & & & & & & & & & $x$ & & & \\
\hline Grewia hirsuta Vahl & $x$ & & $\mathrm{x}$ & $\mathrm{x}$ & $\mathrm{x}$ & $x$ & & $\mathrm{x}$ & $\mathrm{x}$ & & & & & $\mathrm{x}$ & $\mathrm{x}$ & & & \\
\hline Grewia damine Gaertn. & $x$ & & & & & & & & & & & & & & & & & \\
\hline Grewia rothii DC. & $\mathrm{x}$ & & & & & & & & & & & & & $\mathrm{x}$ & & & & \\
\hline Grewia tiliifolia Vahl & $x$ & & & & $x$ & & & & & & & & & $\mathrm{x}$ & & & & \\
\hline Helicteres isora L. & & $\mathrm{x}$ & & & & & & & & & & & & & & & & $x$ \\
\hline Hibiscus panduriformis Burm.f. & $x$ & & & & $\mathrm{x}$ & $x$ & & & & & & & & $\mathrm{x}$ & & & & \\
\hline Kavalama urens (Roxb.) Raf. & $x$ & & & & & & & & & & & & & $\mathrm{x}$ & & & & \\
\hline Kydia calycina Roxb. & $x$ & & & & & & & & & & & & & & & & & \\
\hline $\begin{array}{l}\text { Pterospermum xylocarpum (Gaertn.) } \\
\text { Santapau \& Wagh }\end{array}$ & $x$ & & & & & & & & & $x$ & & & & $x$ & & & & \\
\hline Sida acuta Burm.f. & $x$ & & & $\mathrm{x}$ & $\mathrm{x}$ & $x$ & & $\mathrm{x}$ & & & & & $x$ & $\mathrm{x}$ & & & & \\
\hline Sida cordata (Burm.f.) Borss. Waalk. & $x$ & & & $\mathrm{x}$ & $\mathrm{x}$ & $x$ & & $\mathrm{x}$ & $\mathrm{x}$ & & & & $\mathrm{x}$ & $\mathrm{x}$ & & & $\mathrm{x}$ & \\
\hline Sida cordifolia L. & & & & & & $x$ & & & $\mathrm{x}$ & & & & & & & & & \\
\hline $\begin{array}{l}\text { Thespesia lampas (Cav.) Dalzell } \\
\text { \& A.Gibson }\end{array}$ & & $\mathrm{x}$ & & & $x$ & & & & & & & & & $\mathrm{x}$ & & & $x$ & $x$ \\
\hline Triumfetta rhomboidea Jacq. & $x$ & & & $x$ & $\mathrm{x}$ & $\mathrm{x}$ & $\mathrm{x}$ & $\mathrm{x}$ & $\mathrm{x}$ & & & $\mathrm{x}$ & $\mathrm{x}$ & $\mathrm{x}$ & & & $\mathrm{x}$ & \\
\hline
\end{tabular}




\begin{tabular}{|c|c|c|c|c|c|c|c|c|c|c|c|c|c|c|c|c|c|c|c|}
\hline \multirow[t]{2}{*}{ Scientific name } & \multicolumn{19}{|c|}{ Villages } \\
\hline & 1 & 2 & 3 & 4 & 5 & 6 & 7 & 8 & 9 & 10 & 1 & 1 & 12 & 13 & 14 & 15 & 16 & 17 & 18 \\
\hline Urena lobata L. & $x$ & & & $x$ & $x$ & $x$ & $\mathrm{x}$ & $x$ & $x$ & & & & $\mathrm{x}$ & $x$ & $\mathrm{X}$ & & & $x$ & \\
\hline Waltheria indica $\mathrm{L}$. & $x$ & & $x$ & $x$ & $x$ & $X$ & & $x$ & $x$ & $x$ & & & & $\mathrm{x}$ & $x$ & $x$ & & $x$ & \\
\hline \multicolumn{20}{|l|}{ MELASTOMATACEAE } \\
\hline Memecylon umbellatum Burm.f. & & & & & $x$ & & & & & & & & & & & & & & \\
\hline $\begin{array}{l}\text { Osbeckia stellata Buch.-Ham. } \\
\text { ex Ker Gawl. }\end{array}$ & $x$ & & & & & & & & & & & & & & & & & & \\
\hline \multicolumn{20}{|l|}{ MELIACEAE } \\
\hline Azadirachta indica A.Juss. & & $x$ & & & & & & & & & & & & & & & & & \\
\hline Soymida febrifuga (Roxb.) A.Juss. & $x$ & & & & & & & & & & & & & & & & & & $x$ \\
\hline \multicolumn{20}{|l|}{ MENISPERMACEAE } \\
\hline Cissampelos pareira L. & $x$ & & & & $x$ & $\mathrm{x}$ & & & $x$ & & & & & & $\mathrm{x}$ & & & $\mathrm{x}$ & \\
\hline Cocculus hirsutus (L.) W.Theob. & & & $x$ & & & $\mathrm{X}$ & & & $x$ & & & & & & & & & & \\
\hline \multicolumn{20}{|l|}{ MORACEAE } \\
\hline Ficus benghalensis $\mathrm{L}$. & $x$ & & & & $\mathrm{X}$ & & & & & & & & & & $\mathrm{X}$ & & & & \\
\hline Ficus hispida L.f. & $x$ & $x$ & $\bar{x}$ & $x$ & $\mathrm{x}$ & $\mathrm{x}$ & $\mathrm{x}$ & $\mathrm{x}$ & $x$ & $x$ & & & & $x$ & $\mathrm{x}$ & $x$ & & $\mathrm{x}$ & $x$ \\
\hline Ficus microcarpa L.f. & & $x$ & & & & & & & & & & & & & & & & & \\
\hline Ficus racemosa L. & & & & & & & & $X$ & & & & & & & & & & & \\
\hline $\begin{array}{l}\text { Ficus tinctoria subsp. gibbosa } \\
\text { (Blume) Corner }\end{array}$ & $x$ & & & & & & & & & & & & & & & & & & \\
\hline Ficus virens Aiton & $x$ & & & & & & & & & & & & & & & & & & \\
\hline Streblus asper Lour. & & & & & & & & & & & & & & & & & & $\mathrm{x}$ & \\
\hline \multicolumn{20}{|l|}{ MORINGACEAE } \\
\hline Moringa pterygosperma Gaertn. & & & & & & & & & & & & & & & $\mathrm{X}$ & & & $\mathrm{x}$ & \\
\hline \multicolumn{20}{|l|}{ MYRTACEAE } \\
\hline Syzygium cumini (L.) Skeels & $x$ & & & & $x$ & $\mathrm{X}$ & $x$ & $\mathrm{X}$ & & & & & $x$ & & $\mathrm{x}$ & & $x$ & $\mathrm{x}$ & \\
\hline \multicolumn{20}{|l|}{ NYCTAGINACEAE } \\
\hline Boerhavia diffusa L. & $x$ & & & $x$ & $\mathrm{X}$ & $\mathrm{X}$ & & $\mathrm{X}$ & $x$ & & & & & $x$ & $\mathrm{x}$ & & & $\mathrm{x}$ & \\
\hline \multicolumn{20}{|l|}{ OCHNACEAE } \\
\hline Ochna obtusata DC. & $x$ & & & & & & & $x$ & & & & & & & & & & & \\
\hline \multicolumn{20}{|l|}{ OLEACEAE } \\
\hline Nyctanthes arbor-tristis L. & $x$ & & & & & & & & & & & & & & & & & & \\
\hline Schrebera swietenioides Roxb. & & & & & & $\mathrm{X}$ & & $x$ & & $x$ & & & & & & & & & \\
\hline \multicolumn{20}{|l|}{ ORCHIDACEAE } \\
\hline Vanda tessellata (Roxb.) Hook. ex G.Don & $\bar{x}$ & & & & $x$ & $\mathrm{x}$ & $\mathrm{x}$ & $\mathrm{x}$ & $\mathrm{x}$ & & & & $x$ & & $\mathrm{x}$ & & & $\mathrm{x}$ & \\
\hline \multicolumn{20}{|l|}{ PAPAVARACEAE } \\
\hline Argemone mexicana L. & & & & & & & & & & & & & & & $\mathrm{x}$ & & & $\mathrm{x}$ & \\
\hline \multicolumn{20}{|l|}{ PHYLLANTHACEAE } \\
\hline Breynia retusa (Dennst.) Alston & & & & & $x$ & & & & & & & & & & & & & & \\
\hline Bridelia montana (Roxb.) Willd. & $x$ & & & $\mathrm{X}$ & $\mathrm{x}$ & $\mathrm{x}$ & $\mathrm{x}$ & $\mathrm{X}$ & & & & & $\mathrm{x}$ & $\mathrm{X}$ & $\mathrm{x}$ & & & & \\
\hline Bridelia retusa (L.) A.Juss. & $\bar{x}$ & & & & & & & & & & & & & & & $x$ & & & \\
\hline
\end{tabular}




\section{Suthari et al. - Intracultural Cognizance of Medicinal Plants of Warangal North Forest Division, Northern Telangana, India}

\begin{tabular}{|c|c|c|c|c|c|c|c|c|c|c|c|c|c|c|c|c|c|c|}
\hline \multirow[t]{2}{*}{ Scientific name } & \multicolumn{18}{|c|}{ Villages } \\
\hline & 1 & 2 & 3 & 4 & 5 & 6 & 7 & 8 & 9 & 10 & 11 & 12 & 13 & 14 & 15 & 16 & 17 & 18 \\
\hline $\begin{array}{l}\text { Cleistanthus collinus (Roxb.) Benth. } \\
\text { ex Hook.f. }\end{array}$ & $\mathrm{X}$ & $x$ & & $x$ & $\mathrm{X}$ & $x$ & $\mathrm{x}$ & $x$ & $x$ & & & $x$ & $x$ & $x$ & & & $\mathrm{X}$ & \\
\hline Glochidion zeylanicum (Gaertn.) A.Juss. & $\mathrm{X}$ & & & & & & & & & & & & & & & & & \\
\hline Phyllanthus amarus Schumach. \& Thonn. & $\mathrm{X}$ & & & $X$ & $\mathrm{X}$ & $\mathrm{X}$ & $\mathrm{X}$ & $X$ & & & & $X$ & $x$ & $X$ & & & & \\
\hline Phyllanthus emblica L. & $\mathrm{X}$ & & $\mathrm{x}$ & & $\mathrm{X}$ & $X$ & & $X$ & & & & & & $\mathrm{X}$ & $\mathrm{X}$ & $x$ & $\mathrm{X}$ & \\
\hline Phyllanthus urinaria L. & $\mathrm{X}$ & & $\mathrm{X}$ & & $\mathrm{X}$ & $X$ & & & & & & & & $X$ & $X$ & & $\mathrm{X}$ & \\
\hline \multicolumn{19}{|l|}{ PLANTAGINACEAE } \\
\hline Scoparia dulcis L. & $\mathrm{X}$ & & & & $\mathrm{X}$ & & & & & & & & & $X$ & & & & \\
\hline \multicolumn{19}{|l|}{ PLUMBAGINACEAE } \\
\hline Plumbago zeylanica L. & & & & & & & & & & & & & & $X$ & & & $\mathrm{X}$ & \\
\hline \multicolumn{19}{|l|}{ POACEAE } \\
\hline Bambusa bambos (L.) Voss & & & & & & & & & & $x$ & & & & $X$ & & & $\mathrm{X}$ & \\
\hline $\begin{array}{l}\text { Cymbopogon flexuosus (Nees ex Steud.) } \\
\text { W.Watson }\end{array}$ & $x$ & & & & & & & & & & & & & & & & & \\
\hline \multicolumn{19}{|l|}{ PUTRANJIVACEAE } \\
\hline Putranjiva roxburghii Wall. & $\mathrm{X}$ & & & & & & & & & & & & & & & & & \\
\hline \multicolumn{19}{|l|}{ RHAMNACEAE } \\
\hline Ventilago maderaspatana Gaertn. & $\mathrm{X}$ & & & & $\mathrm{X}$ & $\mathrm{X}$ & $\mathrm{X}$ & $\mathrm{X}$ & $\mathrm{X}$ & & & & & $\mathrm{X}$ & & & $\mathrm{X}$ & \\
\hline Ziziphus oenopolia (L.) Mill. & & & & & & & & & & & & & & & & & $\mathrm{X}$ & \\
\hline Ziziphus rugosa Lam. & $x$ & & & & & & & & & & & & & & & & & \\
\hline Ziziphus xylopyrus (Retz.) Willd. & $\mathrm{X}$ & & $\mathrm{X}$ & & $\mathrm{X}$ & $\mathrm{X}$ & $\mathrm{X}$ & $\mathrm{X}$ & $x$ & & & $x$ & & $\mathrm{X}$ & $\mathrm{X}$ & & $x$ & \\
\hline \multicolumn{19}{|l|}{ RUBIACEAE } \\
\hline Catunaregam spinosa (Thunb.) Tirveng. & & & $\mathrm{X}$ & & & & & & & & & & & & $x$ & & & $x$ \\
\hline Ceriscoides turgida (Roxb.) Tirveng. & & & & & $\mathrm{X}$ & & & & & & & & & $X$ & & & $\mathrm{X}$ & \\
\hline Gardenia latifolia Aiton & $x$ & & $\mathrm{X}$ & & $\mathrm{x}$ & $\mathrm{X}$ & & $\mathrm{X}$ & & & & & & & $\mathrm{X}$ & $\mathrm{X}$ & $x$ & \\
\hline Gardenia resinifera Roth & $\mathrm{X}$ & & & $\mathrm{X}$ & $\mathrm{X}$ & $\mathrm{X}$ & & $\mathrm{X}$ & & $X$ & & & $x$ & & $x$ & & & \\
\hline Haldina cordifolia (Roxb.) Ridsdale & $\mathrm{X}$ & & & & $\mathrm{x}$ & $\mathrm{X}$ & & & $\mathrm{x}$ & & & & & $\mathrm{X}$ & & & & \\
\hline Ixora arborea Roxb. ex Sm. & $x$ & & $x$ & & $x$ & $x$ & $x$ & $\mathrm{X}$ & $x$ & $x$ & & & & $x$ & $x$ & & $x$ & \\
\hline Mitragyna parvifolia (Roxb.) Korth. & & & & & & $\mathrm{X}$ & & $\mathrm{X}$ & & & & & & & & & & \\
\hline Morinda pubescens Sm. & $\mathrm{x}$ & $x$ & $x$ & $\mathrm{x}$ & $\mathrm{x}$ & $\mathrm{X}$ & $x$ & $x$ & $x$ & & & $x$ & $x$ & $\mathrm{x}$ & $x$ & & $x$ & \\
\hline Paederia foetida $\mathrm{L}$. & $\mathrm{X}$ & & & & & & & & & & & & & & & & & \\
\hline $\begin{array}{l}\text { Tamilnadia uliginosa (Retz.) Tirveng. } \\
\text { \& Sastre }\end{array}$ & $x$ & & & & & & & & & & & & & & & & & \\
\hline \multicolumn{19}{|l|}{ RUTACEAE } \\
\hline Aegle marmelos (L.) Corrêa & $\mathrm{X}$ & $\mathrm{X}$ & & & $\mathrm{X}$ & $\mathrm{X}$ & & $\mathrm{X}$ & $\mathrm{X}$ & & $X$ & & & $\mathrm{X}$ & & $x$ & $\mathrm{X}$ & \\
\hline Atalantia monophylla DC. & & & & & & & & & & & & & & & $\mathrm{X}$ & & & \\
\hline Chloroxylon swietenia DC. & $\mathrm{X}$ & & & $\mathrm{X}$ & $\mathrm{X}$ & $\mathrm{X}$ & $\mathrm{X}$ & $\mathrm{X}$ & $x$ & & $\mathrm{X}$ & $\mathrm{X}$ & $x$ & $\mathrm{X}$ & & & $\mathrm{X}$ & \\
\hline Limonia acidissima L. & & $\mathrm{X}$ & & & & & & & & & & & & & & & & \\
\hline \multicolumn{19}{|l|}{ SALICACEAE } \\
\hline Casearia nigrescens Tue. & $x$ & & & & $x$ & $x$ & & & & & & & & & & & & \\
\hline Flacourtia indica (Burm.f.) Merr. & $x$ & & & & $x$ & & & & & & & & & $x$ & & & $x$ & $x$ \\
\hline
\end{tabular}




\begin{tabular}{|c|c|c|c|c|c|c|c|c|c|c|c|c|c|c|c|c|c|c|}
\hline \multirow[t]{2}{*}{ Scientific name } & \multicolumn{18}{|c|}{ Villages } \\
\hline & 1 & 2 & 3 & 4 & 5 & 6 & 7 & 8 & 9 & 10 & \begin{tabular}{|l|l|l|}
11 & \\
\end{tabular} & 12 & 13 & 14 & 15 & 16 & 17 & 18 \\
\hline \multicolumn{19}{|l|}{ SAPINDACEAE } \\
\hline Sapindus emarginatus Vahl & & & & & & & & & & & & & & & & $x$ & & \\
\hline Schleichera oleosa (Lour.) Merr. & $\mathrm{X}$ & & & & & & & & & & & & & & & & $x$ & \\
\hline \multicolumn{19}{|l|}{ SAPOTACEAE } \\
\hline $\begin{array}{l}\text { Madhuca longifolia var. latifolia (Roxb.) } \\
\text { A.Chev. }\end{array}$ & $x$ & $x$ & & $x$ & $x$ & $x$ & $x$ & $x$ & $x$ & & & $x$ & $x$ & $\mathrm{x}$ & $x$ & & $x$ & \\
\hline Manilkara hexandra (Roxb.) Dubard & & & & & $x$ & & & & & & & & & & & & & \\
\hline \multicolumn{19}{|l|}{ SIMAROUBACEAE } \\
\hline Ailanthus excelsa Roxb. & & & & & & & & & & & & & & & & $x$ & & \\
\hline \multicolumn{19}{|l|}{ SMILACACEAE } \\
\hline Smilax perfoliata Lour. & $\mathrm{X}$ & & & & & & & & & & & & & & & & & \\
\hline \multicolumn{19}{|l|}{ SOLANACEAE } \\
\hline Solanum surattense Burm.f. & $\mathrm{X}$ & & & $\mathrm{x}$ & $\mathrm{x}$ & $x$ & $\mathrm{X}$ & $x$ & $\mathrm{x}$ & & & $\mathrm{X}$ & $\mathrm{x}$ & $x$ & & & $x$ & \\
\hline \multicolumn{19}{|l|}{ ULMACEAE } \\
\hline Holoptelea integrifolia Planch. & $\mathrm{X}$ & & & & $x$ & $x$ & $\mathrm{x}$ & $x$ & $\mathrm{x}$ & $x$ & $x$ & & & $x$ & $\mathrm{x}$ & & & \\
\hline \multicolumn{19}{|l|}{ VIOLACEAE } \\
\hline Hybanthus enneaspermus (L.) F.Muell. & $\mathrm{X}$ & & $\mathrm{X}$ & $\mathrm{x}$ & $\mathrm{x}$ & $x$ & $\mathrm{x} \mid$ & $x$ & $x$ & & & $x$ & $x$ & $x$ & $x$ & & $x$ & \\
\hline \multicolumn{19}{|l|}{ VITACEAE } \\
\hline Cissus vitiginea $\mathrm{L}$. & & & & & & $x$ & & $x$ & & & & & & & & $\mathrm{X}$ & & \\
\hline Leea asiatica (L.) Ridsdale & & & & & & & & & & $x$ & $x$ & & & & $x$ & & & \\
\hline \multicolumn{19}{|l|}{ ZINGIBERACEAE } \\
\hline Curcuma pseudomontana J.Graham & $x$ & & & & & & & & & & & & & & & & & \\
\hline TOTAL SPECIES RICHNESS & 167 & 19 & 28 & 42 & 107 & 105 & 34 & 68 & 59 & 21 & 21 & 25 & 36 & 105 & 40 & 15 & 86 & 21 \\
\hline
\end{tabular}

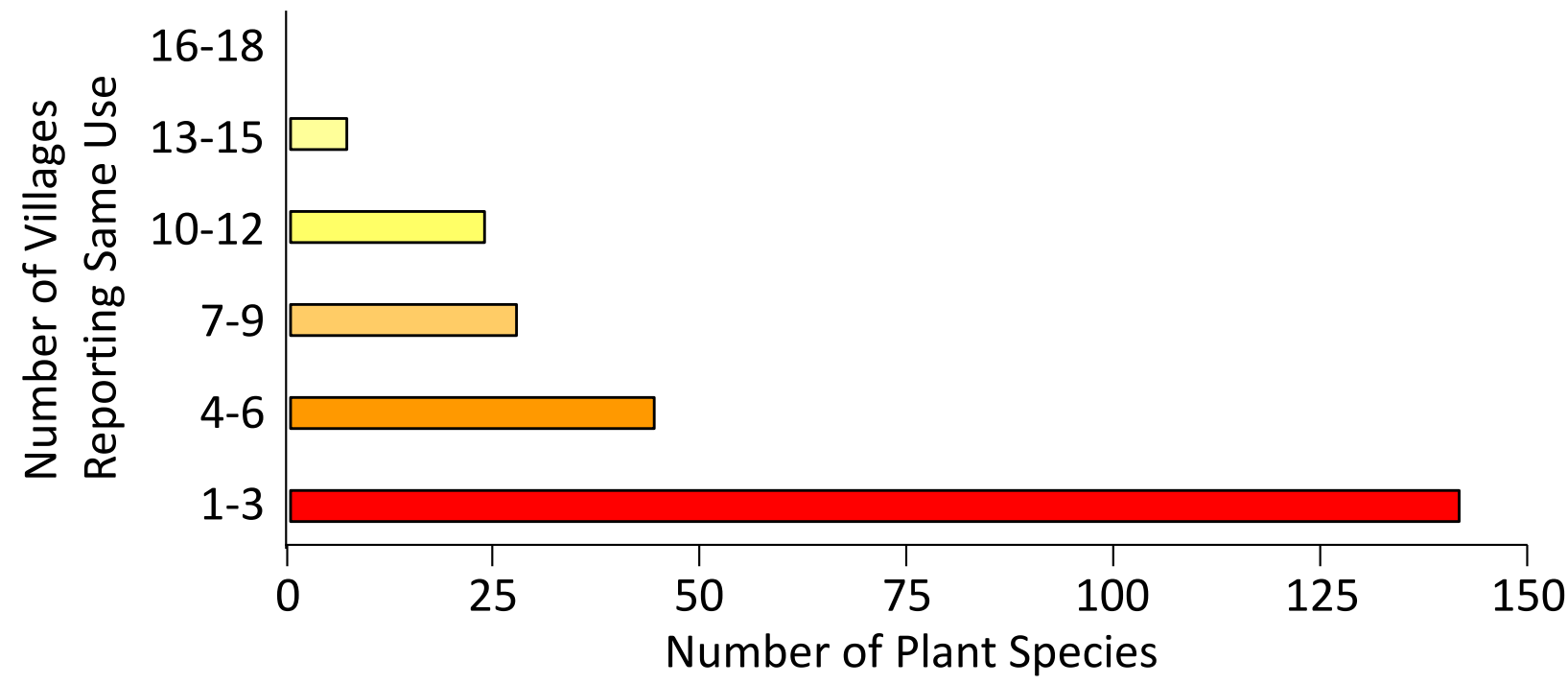

Figure 2. Knowledge sharing among inhabitants of 18 Koya villages within or near Eturnagaram Wildlife Sanctuary in Warangal North Forest Division of Northern Telangana, India. Note that no shared plant species were reported by 16 or more villages. 


\section{Suthari et al. - Intracultural Cognizance of Medicinal Plants of Warangal North Forest Division, Northern Telangana, India}

(Table 5). The plant families, in the order of their utility, are Fabaceae, Malvaceae, Euphorbiaceae s.I., Rubiaceae, and Acanthaceae. Of these, Fabaceae (39 species) is the only family which is put to use in all 18 villages. It is followed by Malvaceae (18 species), Euphorbiaceae s.l. (15 species), Rubiaceae (10 species), and Acanthaceae (9 species). When the total family-use values of villages are viewed, three of them (Shapally, Dodla, and Narlapur) form a triangle within the sanctuary, with high top five family-use values totaling $2.43,2.24$, and 2.16 , respectively. The least family-use value among the 16 villages in Eturnagaram Wildlife Sanctuary is Oorattum, with the value 0.15 . The two villages located outside the sanctuary and used here to compare have the dominant five familyuse values totaling 3.44 for Mallur, which is high, and 0.35 for Gandhinagar, which is very low compared to both the mean value of all villages (1.17) and the within-sanctuary mean (1.08).

\section{Discussion}

The dominant families of ethnic use reported are all dicots, supporting the assertion by Gottlieb et al. (2002) that dicotyledonous families possess more medicinal properties. Differential use of plant taxa according to class rank may be due to knowledge of the prevalence of bioactive compounds and/or the simple preponderance of these taxa in the foraging area. Obviously, the ethnic knowledge of medicinal plants of Koya community is variable and is not uniformly spread amongst either the informants/inhabitants or the floristic groups.

Another observation, though not experimentally demonstrated here, is that the local people knew of more medicinal plants than they actually used. When local people were asked to mention names and uses other than those listed; they recalled some species but declared that they no longer use them. The village-wise difference in num- ber of plant species used by the Koyas living in the same habitat vouches for this. The intracultural differences in the knowledge domain could be due to other available medical options or erosion of traditional pharmacological knowledge.

The patterns of medicinal plant use by local people are considered to vary as a function of plant habitat diversity, cultural changes, ecological, and biochemical aspects (Stepp 2004). Besides, the diversity in the ecological knowledge held by individuals within indigenous communities, as in Koyas, may be fundamental to community adaptation. For example, Standing Rock elders (Ruelle \& Kassam 2011) themselves challenged that their knowledge is not homogeneous within their community. The variables that have been found to pattern the intracultural variation of ethnobotanical knowledge are age (Phillips \& Gentry 1993), gender, kinship (Boster 1986), acculturation (Zent 2001), and level of integration with the market economy (Reyes-Garcia 2001). The conscious perception of cognitive signals, together with the individual cultural history of different ethnic groups, assert that these signals may be connected and interpreted in culture-specific ways.

\section{Acknowledgments}

The authors thank all the ethnic informants in the study area who shared their traditional botanical knowledge of healthcare; Prof. A. Ragan, Head, Department of Botany, Kakatiya University, Warangal, Andhra Pradesh, for facilities under SAP program; and Dr. J. Srinivasa Rao, Department of Statistics, Kakatiya University, for the help with ANOVA.

Table 5. Family-use values of the five most ethnomedicinally important plant families as reported by Koya inhabitants of 18 villages within or near Eturnagaram Wildlife Sanctuary in Warangal North Forest Division of Northern Telangana, India. Villages 1 and 18 are outside the sanctuary. Villages: $1=$ Mallur; $2=$ Kamaram; $3=$ Venkatapuram; $4=$ Chinnaboinapally; 5 = Shapally; 6 = Dodla; $7=$ Malyala; $8=$ Kondai; $9=$ Ailapur; $10=$ Yelishettipally; $11=$ Banojibandam; $12=$ Chalpaka; 13 = Allamvari Ghanpur; 14 = Narlapur; 15 = Medaram; 16 = Oorattum; 17 = Gonepally; $18=$ Gandhinagar. * The family Euphorbiaceae here includes Phyllanthaceae and Putranjivaceae.

\begin{tabular}{|c|c|c|c|c|c|c|c|c|c|c|c|c|c|c|c|c|c|c|}
\hline \multirow[t]{2}{*}{ Family } & \multicolumn{18}{|c|}{ Villages } \\
\hline & 1 & 2 & 3 & 4 & 5 & 6 & 7 & 8 & 9 & 10 & 11 & 12 & 13 & 14 & 15 & 16 & 17 & 18 \\
\hline Fabaceae & 0.73 & 0.05 & 0.08 & 0.15 & 0.44 & 0.40 & 0.13 & 0.21 & 0.12 & 0.10 & 0.21 & 0.05 & 0.13 & 0.49 & 0.10 & 0.05 & 0.41 & 0.10 \\
\hline Malvaceae & 0.72 & 0.14 & 0.11 & 0.33 & 0.47 & 0.50 & 0.11 & 0.33 & 0.39 & 0.08 & 0.00 & 0.11 & 0.28 & 0.67 & 0.14 & 0.00 & 0.33 & 0.08 \\
\hline Euphorbiaceae s.l.* & 0.50 & 0.07 & 0.07 & 0.17 & 0.37 & 0.23 & 0.10 & 0.17 & 0.13 & 0.07 & 0.07 & 0.10 & 0.17 & 0.17 & 0.37 & 0.00 & 0.13 & 0.07 \\
\hline Rubiaceae & 0.60 & 0.10 & 0.30 & 0.25 & 0.60 & 0.55 & 0.20 & 0.50 & 0.25 & 0.25 & 0.00 & 0.10 & 0.25 & 0.40 & 0.40 & 0.10 & 0.40 & 0.10 \\
\hline Acanthaceae & 0.89 & 0.00 & 0.00 & 0.22 & 0.56 & 0.56 & 0.11 & 0.33 & 0.22 & 0.00 & 0.00 & 0.11 & 0.22 & 0.44 & 0.00 & 0.00 & 0.44 & 0.00 \\
\hline TOTAL & 3.44 & 0.36 & 0.55 & 1.13 & 2.43 & 2.24 & 0.65 & 1.54 & 1.11 & 0.50 & 0.27 & 0.47 & 1.04 & 2.16 & 1.01 & 0.15 & 1.72 & 0.35 \\
\hline
\end{tabular}




\section{Literature Cited}

Anonymous. 2011a. Census Report. Government of Andhra Pradesh, Hyderabad, India.

Anonymous. 2011b. Andhra Pradesh State of Forest Report. Andhra Pradesh Forest Department, Government of Andhra Pradesh, Hyderabad, India.

Boster, J.S. 1986. Exchange of varieties and information between Aguaruna manioc cultivators. American Anthropologist 88(2):428-436. dx.doi.org/10.1525/ aa.1986.88.2.02a00100

Carlson,T.J., B.M. Foula, J.A. Chinnock, S.R. King, G. Abdourahmaue, B.M. Sannoussy, A. Bah, S.A. Cisse \& R.K. Richter. 2001. Case study on medicinal plant research in guinea: Prior informed consent, focused benefit sharing, and compliance with the Convention on Biological Diversity. Economic Botany 55(4):478-491. dx.doi.org/10.1007/ BF02871712

Gamble, J.S. \& C.E.C. Fischer. 1915-1935. Flora of the Presidency of Madras. Volumes I-III. Adlord \& Sons Ltd., London, U.K.

Gottlieb, O.R., M.R. de M.B. Borin \& N.R.S. de Brito. 2002. Integration of ethnobotany and phytochemistry: Dream or reality? Phytochemistry 60(2):145-152. dx.doi. org/10.1016/S0031-9422(02)00088-2

Harmon, D. 2002. In Light of our Differences: How diversity in nature and culture makes us human. Smithsonian Institution Scholarly Press, Washington, D.C., U.S.A.

Hoffman, B. \& T. Gallaher. 2007. Importance indices in ethnobotany. Ethnobotany Research \& Applications 5:201-218. lib-ojs3.lib.sfu.ca:8114/index.php/era/article/ view/130

Kassam, K.-A. 2009. Biocultural Diversity and Indigenous Ways of Knowing: Human ecology in the Arctic. University of Calgary Press, Calgary, Alberta, Canada.

Murthy, E.N., C.S. Reddy, K.N. Reddy \& V.S. Raju. 2007. Plants used in ethnoveterinary practices by Koyas of Pakhal Wildlife Sanctuary, Andhra Pradesh, India. Ethnobotanical Leaflets 11:1-5. www.ethnoleaflets.com/ leaflets/reddy2.htm

Phillips, O. \& A.H. Gentry. 1993. The useful plants of Tambopata, Peru: II. Additional hypothesis testing in quantitative ethnobotany. Economic Botany 47(1):33-43. dx.doi. org/10.1007/BF02862204

Raju, V.S. 2013. Ethnobotany: Retrospect and prospect. Pp. 5-7 in Proceedings of the National Seminar on Genomes, Environment and Human Welfare: Retrospect and prospect. 22-23 March, Andhra University, Waltair, Visakhapatnam, Andhra Pradesh, India.

Rao, N.R. 1988. The Ethnobotany of Eastern Ghats in Andhra Pradesh, India. Ph.D. Dissertation, Bharathiar University, Coimbatore, Tamil Nadu, India.

Rao, N.R. \& A.N. Henry. 1996. The Ethnobotany of Eastern Ghats in Andhra Pradesh, India. Botanical Survey of India, Kolkata, West Bengal, India.

Reddy, C.S., P.G. Krishna \& V.S. Raju. 2008. Phytotherapy at rural communities: A case study from the Gonds of Warangal District, Andhra Pradesh, India. Research Journal of Botany 3(2):97-102.

Reddy, K.N., M.R. Bhanja \& V.S. Raju. 1998. Plants used in ethnoveterinary practices in Warangal District, Andhra Pradesh, India. Ethnobotany 10(1-2):75-84.

Reyes-Garcia, V. 2001. Indigenous People, Ethnobotanical Knowledge, and Market Economy. A case study of the Tsimane Amerindians in Lowland Bolivia. Ph.D. Dissertation, University of Florida, Gainsville, Florida, U.S.A.

Romney, A.K., S.C. Weller \& W.H. Batchelder. 1986. Culture as consensus: A theory of culture and informant accuracy. American Anthropologist 88(2):313-338. dx.doi. org/10.1525/aa.1986.88.2.02a00020

Rosenthal, J.P. 2006. Politics, culture, and governance in the development of prior informed consent in indigenous communities. Current Anthropology 47(1):119-142. dx.doi.org/10.1086/497670

Ruelle, M.L. \& K.-A.S. Kassam. 2011. Diversity of plant knowledge as an adaptive asset: A case study with Standing Rock elders. Economic Botany 65(3):295-307. dx.doi.org/10.1007/s12231-011-9168-x

Singh, R.K. 2008. Implications of prior informed consent for the conservators of indigenous biological diversity of Northeast India. Indian Journal of Traditional Knowledge 7(4):655-665. nopr.niscair.res.in/handle/123456789/2387

Sreeramulu, N. 2009. Traditional Botanical Knowledge of Local People in Nalgonda and Warangal Districts of Telangana, Andhra Pradesh, India. Ph.D. Dissertation, Kakatiya University, Warangal, Andhra Pradesh, India.

Stepp, J.R. 2004. The role of weeds as sources of pharmaceuticals. Journal of Ethnopharmacology 92(2-3):163166. $\underline{\mathrm{dx} . \mathrm{doi} . \mathrm{org} / 10.1016 / \mathrm{j} . j \mathrm{jep} .2004 .03 .002}$

Turner, N.J. \& H. Clifton. 2009. "It's so different today": Climate change and indigenous lifeways in British Colombia, Canada. Global Environmental Change 19(2):180-190. dx.doi.org/10.1016/j.gloenvcha.2009.01.005 


\section{Suthari et al. - Intracultural Cognizance of Medicinal Plants of Warangal North Forest Division, Northern Telangana, India}

Zent, S. 2001. Acculturation and ethnobotanical knowledge loss among the Piaroa of Venezuela: Demonstration of a quantitative method for the empirical study of Traditional Ecological Knowledge change Pp. 190-211 in On Biocultural Diversity: Linking language, knowledge, and the environment. Edited by L. Maffi. Smithsonian Institution Press, Washington, D.C., U.S.A. 
